\title{
Evidencias geológicas, geomorfológicas y geofísicas de deformación asociada a la falla Cerritos y su implicación en el peligro sísmico de Morelia, Michoacán, México
}

\author{
Diana Cinthia Soria-Caballero' ${ }^{1}$, Martha Gabriela Gómez-Vasconcelos ${ }^{2 *}$, \\ Denis Ramón Avellán ${ }^{3}$, Jélime Aray Castellano ${ }^{4}$, Dulce Gutiérrez-Carmona ${ }^{5}$, \\ Adrián Jiménez-Haro ${ }^{5}$, Magdalena Velázquez-Bucio ${ }^{6}$, Jesús Ávila García ${ }^{7}$, \\ Oscar García ${ }^{5}$ y Víctor Hugo Garduño-Monroy ${ }^{5, \dagger}$
}

${ }^{1}$ Escuela Nacional de Estudios Superiores, Unidad Morelia, Universidad Nacional Autónoma de México, Antigua Carretera a Pátzcuaro 8701, Ex-Hacienda de San José de La Huerta, 58190, Morelia, Michoacán, México.

${ }^{2}$ CONACYT-Instituto de Investigaciones en Ciencias de la Tierra, Universidad Michoacana de San Nicolás de Hidalgo, Santiago Tapia 403, 58000, Morelia, Michoacán, México.

${ }^{3}$ CONACYT-Instituto de Geofísica Unidad Michoacán, Universidad Nacional Autónoma de México, Antigua Carretera a Pátzcuaro 8701, 58190, Morelia, Michoacán, México.

${ }^{4}$ Posgrado en Ciencias de la Tierra, Centro de Geociencias, Universidad Nacional Autónoma de México, Blvd. Juriquilla, 3001, 76230, Querétaro, México.

${ }^{5}$ Instituto de Investigaciones en Ciencias de la Tierra, Universidad Michoacana de San Nicolás de Hidalgo, Santiago Tapia 403, 58000, Morelia, Michoacán, México.

${ }^{6}$ Dipartimento di Scienza e Alta Tecnologia, Università degli Studi dell'Insubria, Via Valleggio 11, 22100, Como, Italia.

${ }^{7}$ Posgrado en Ciencias de la Tierra, Instituto de Geofísica, Universidad Nacional Autónoma de México,

Circuito de la Investigación Científica, Ciudad Universitaria, Ciudad de México, 04510, México.

*gabriela.gomez@umich.mx

\section{RESUMEN}

La falla Cerritos pertenece a la porción oeste del Sistema de Fallas Morelia-Acambay, ubicada al suroeste de la ciudad de Morelia, en el estado de Michoacán, México; es una zona con alta densidad de fallas activas con geometrías segmentadas y arreglos en échelon, donde se han desarrollado semi-grabens y grabens de dimensiones variables que inclusive influyen en el alineamiento y distribución del vulcanismo monogenético holocénico. En la superficie, estas fallas afectan unidades litológicas de edades miocénicas hasta suelos modernos. Debido a la peligrosidad sísmica que se ha reportado para algunas de las fallas de Morelia, se realizó un análisis interdisciplinario de la falla Cerritos, que había permanecido poco estudiada hasta la fecha. La falla Cerritos es una falla normal con una ligera componente lateral izquierda, una longitud de $12 \mathrm{~km}$ y un relieve topográfico bien definido de $130 \pm 5 \mathrm{~m}$ (100 $\mathrm{m}$ de desplazamiento visible $+30 \mathrm{~m}$ de desplazamiento subsuperficial obtenido por el estudio de resistividad invertida). Esta falla muestra un rumbo ENE-OSO $\left(255^{\circ}\right)$ y un echado de $76^{\circ}$ hacia el NNO. En un afloramiento de la falla Cerritos, a unos cuantos metros del trazo de falla principal, se identificó una cuña coluvial con abundante materia orgánica, fechada con el método de radiocarbono en 3.37-3.21 cal ka AP. El análisis geológico y geomorfológico de la falla reveló que es una estructura tectónicamente activa, relativamente joven y con índices geomórficos que indican mayor actividad en su sector oriental. El escarpe y la acumulación de sedimentos lacustres en el bloque caído sugieren movimiento activo vertical (levantamiento y subsidencia, respectivamente) a lo largo de esta falla. Los resultados obtenidos mediante los análisis de sísmica de refracción, tomografía de resistividad eléctrica y magnetometría terrestre revelaron la geometría de la falla en profundidad, en donde sobresale la presencia de un plano principal lístrico y una zona de deformación en el bloque caído que se extiende en superficie hasta $75 \mathrm{~m}$ desde el pie del escarpe principal. Esta zona de deformación se caracteriza por la formación de anticlinales tipo roll-over y dos grabens de colapso crestal delimitados por estructuras secundarias, los cuales funcionan como depocentros de materiales clásticos y bloques caídos. Finalmente, el análisis de actividad y potencialidad indicó que esta estructura tiene tasas de desplazamiento de $0.03 \pm 0.01 \mathrm{~mm} / \mathrm{año}$, con desplazamientos verticales promedio de $0.5 \mathrm{~m}$ por evento, $\mathrm{y}$ un tiempo de recurrencia promedio de 16700 años. La Falla Cerritos tiene la capacidad de producir eventos de ruptura con magnitudes de $M_{\mathrm{W}} 6.2$ a 6.6 por sí sola, o bien, puede interaccionar en un evento extremo con las fallas adyacentes Morelia y Cointzio, produciendo eventos de ruptura multi-falla con magnitudes de hasta $M_{\mathrm{W}} 6.9$.

Palabras clave: falla activa; índice geomórfico;paleosismología; resistividad eléctrica; sísmica de refracción; sistema de fallas MoreliaAcambay; México.

Soria-Caballero, D.C., Gómez-Vasconcelos, M.G., Avellán, D.R., Aray Castellano, J., Gutiérrez-Carmona, D., Jiménez-Haro, A., Velázquez-Bucio, M.M., Ávila García, J., García, O., Garduño-Monroy, V.H., 2021, Evidencias geológicas, geomorfológicas y geofísicas de deformación asociada a la falla Cerritos y su implicación en el peligro sísmico de Morelia, Michoacán, México: Revista Mexicana de Ciencias Geológicas, v. 38, núm. 3, p. 239-258. 


\section{ABSTRACT}

The Cerritos fault is located SW of Morelia city, in the state of Michoacán, Mexico. The fault belongs to the western part of the MoreliaAcambay Fault System, an area with several active segmented faults that form various grabens and half-grabens. In this area, faulting affects Miocene to Holocene lithologies; some of these faults even control the distribution of regional monogenetic volcanoes. This work is an interdisciplinary approach to characterize the Cerritos fault, an important structure with a high seismic hazard potential. The Cerritos fault is a $12 \mathrm{~km}$ long oblique fault (normal-left lateral) with a $130 \pm 5 \mathrm{~m}$-high topographic relief (100 $\mathrm{m}$ of surface displacement $+30 \mathrm{~m}$ of subsurface displacement estimated from the inverted resistivity study). This fault is ENE-WSW oriented $\left(255^{\circ}\right)$, with a $76^{\circ} \mathrm{NNW}$ dip. In a well-exposed cross section of the Cerritos fault, a few meters from the master fault trace, a colluvial wedge with organic material was identified, yielding a radiocarbon age of 3.37-3.21 cal ka BP. Our geologic and geomorphological analyses indicate that the Cerritos fault is a young, tectonically-active fault, especially in its eastern part. The vertical and horizontal linearity of the fault scarp and the accumulation of lake deposits in the down-thrown block suggest active vertical motion (uplift and subsidence, respectively) along this fault. Geophysical surveys, including seismic refraction, terrestrial magnetometry, and electrical resistivity tomography, show the subsurface geometry of the fault to be characterized by a main listric fault plane and a damage zone in the footwall block, extending as far as $75 \mathrm{~m}$ from the main scarp. The damage zone is characterized by secondary, synthetic, and antithetic faults, forming roll-over anticlines and two crestal collapse grabens that accumulate colluvial material. Paleoseismic estimates of activity and seismic hazard potential indicate that the Cerritos fault has a slip-rate of $0.03 \pm 0.01 \mathrm{~mm} / \mathrm{yr}$, with mean vertical displacements of $0.5 \mathrm{~m}$ per event and a mean recurrence interval of 16700 years. The Cerritos fault can generate single-segment ruptures with magnitudes of $M_{W} 6.2$ to 6.6. Still, in a worst-case scenario, it could also rupture with the subparallel and adjacent Morelia and Cointzio faults, generating earthquake magnitudes up to $M_{W} 6.9$.

Key words: Active fault; geomorphic indicator;paleoseismicity; seismic refraction; electrical resistivity tomography; Morelia-Acambay fault system; Mexico.

\section{INTRODUCCIÓN}

México es un país frecuentemente afectado por sismos tanto de subducción como continentales y, en menor medida, por los producidos por actividad volcánica. Aunque los sismos de subducción han recibido más atención, los sismos continentales son igualmente importantes, pues tienen la capacidad de generar cuantiosos daños a pesar de tener magnitudes moderadas (p.ej., Suter, 2017). Esto ha sido particularmente evidente para las poblaciones ubicadas en el centro del país, como la Ciudad de México, los estados de México, Puebla, Michoacán y Jalisco, entre otras, las cuales son afectadas por sistemas de fallas creados durante la formación y desarrollo del Cinturón Volcánico Trans-Mexicano (CVTM).

Desde hace aproximadamente 100 años, se ha considerado que en los sistemas de fallas del centro de México se ubican fallas activas que se han movido en los últimos 10000 años y que tienen potencial para generar sismos importantes en el futuro (Comisiones del Instituto Geológico de México, 1922; Camacho, 1925; Mendoza-Ponce et al., 2018). Inclusive la geomorfología de la región parece ser resultado de actividad sísmica repetida durante miles o millones de años (Suter et al., 1995 y 2001; Ego y Ansan, 2002; Andreani et al., 2008). Entre estos sistemas se incluye el Sistema de Fallas Morelia-Acambay (SFMA) que ha mostrado su potencialidad al ser la fuente de varios sismos importantes (p.ej., sismo de Acambay en 1912, $M_{\mathrm{W}}$ 7.0; Urbina y Camacho, 1913; Langridge et al., 2000).

La ciudad de Morelia, ubicada al norte de Michoacán, México, es una zona severamente afectada por fallamiento continental asociado al SFMA, así como por procesos relacionados a la subsidencia y creep, los cuales han ocasionado daños importantes en la infraestructura de la ciudad (Garduño-Monroy et al., 2001; Ávila-Olivera y GarduñoMonroy, 2008; Farina et al., 2008; Cigna et al., 2012; Figueroa-Miranda et al., 2020). Al ser la capital del estado, Morelia concentra una población de 988704 habitantes en su área metropolitana, distribuidos en una superficie de alrededor de $1200 \mathrm{~km}^{2}$. Esta ciudad cuenta con una tasa de crecimiento relativamente alta, de $1.77 \%$ anual (INEGI, 2020), evidente en el aumento de la mancha urbana y la demanda de servicios. Este incremento se ve reflejado en la creación de conjuntos habitacionales y otras infraestructuras necesarias que suele carecer de análisis geológicos y geofísicos que permitan evaluar la idoneidad de los sitios donde se construye. Considerando la peligrosidad sísmica de la región, esto puede convertirse en un problema de seguridad a largo plazo y ocasionar cuantiosas pérdidas materiales.

El presente trabajo se enfoca en la caracterización geológica, geomorfológica y geofísica de la falla Cerritos ubicada al suroeste de Morelia; una falla con orientación ENE-OSO y un escarpe bien definido (Figura 1). La falla Cerritos pertenece a la porción occidental del SFMA y, aunque no es una de las estructuras más grandes de la zona, su ubicación en la ciudad de Morelia, su cercanía a otras estructuras y su orientación con respecto al campo de esfuerzos actual denotan su peligrosidad sísmica. El escarpe y la acumulación de sedimentos lacustres en el bloque caído sugieren movimiento activo vertical (levantamiento y subsidencia, respectivamente) a lo largo de esta falla; sin embargo, su potencial sismogenético no ha sido evaluado en detalle. La caracterización aquí presentada contribuye a mejorar el entendimiento de la potencialidad sísmica de esta estructura dentro del contexto neotectónico de la región.

\section{MARCO GEOLÓGICO Y TECTÓNICO}

La ciudad de Morelia se localiza en el sector central del CVTM, un arco magmático de edad miocénica-holocénica que atraviesa el centro del país, desde las costas de Jalisco y Nayarit en el Océano Pacífico hasta las costas de Veracruz en el Golfo de México (Figura 1; Demant, 1978; Ferrari et al., 2012; Gómez-Tuena et al., 2005). El CVTM es una de las provincias geológicas más importantes de México, tanto desde el punto de vista geológico como social. Presenta una geometría oblicua respecto a la Trinchera Mesoamericana, la cual se explica por las variaciones en las velocidades, ángulos de subducción y geometrías de las placas Rivera y Cocos moviéndose debajo de la placa de Norteamérica (Pardo y Suárez, 1995; Manea et al., 2013; Guzmán-Speziale y Zúñiga, 2016).

El basamento local en el área de estudio está representado por las lavas andesitas basálticas Cuitzeo del Mioceno temprano (18.6818.31 Ma; Avellán et al., 2020) que afloran en la parte este de la falla Morelia (Figura 2). Están sobreyacidas por la ignimbrita Cuitzeo con una edad de 17.4 Ma (Figura 2; Avellán et al., 2020). A su vez, la ignimbrita Cuitzeo es sobreyacida por la ignimbrita Atécuaro (antes denominada ignimbrita Morelia por Pasquarèet al., 1991) de $16.4 \mathrm{Ma}$ de edad (Gómez-Vasconcelos et al., 2015) y su formación se asocia al colapso de la caldera con el mismo nombre. La ignimbrita Atécuaro está ampliamente expuesta en toda la región sur de Morelia, y la ciudad se encuentra asentada en parte sobre estos depósitos. Esta unidad ha sido cubierta parcialmente en varios sectores al noroeste de la caldera 


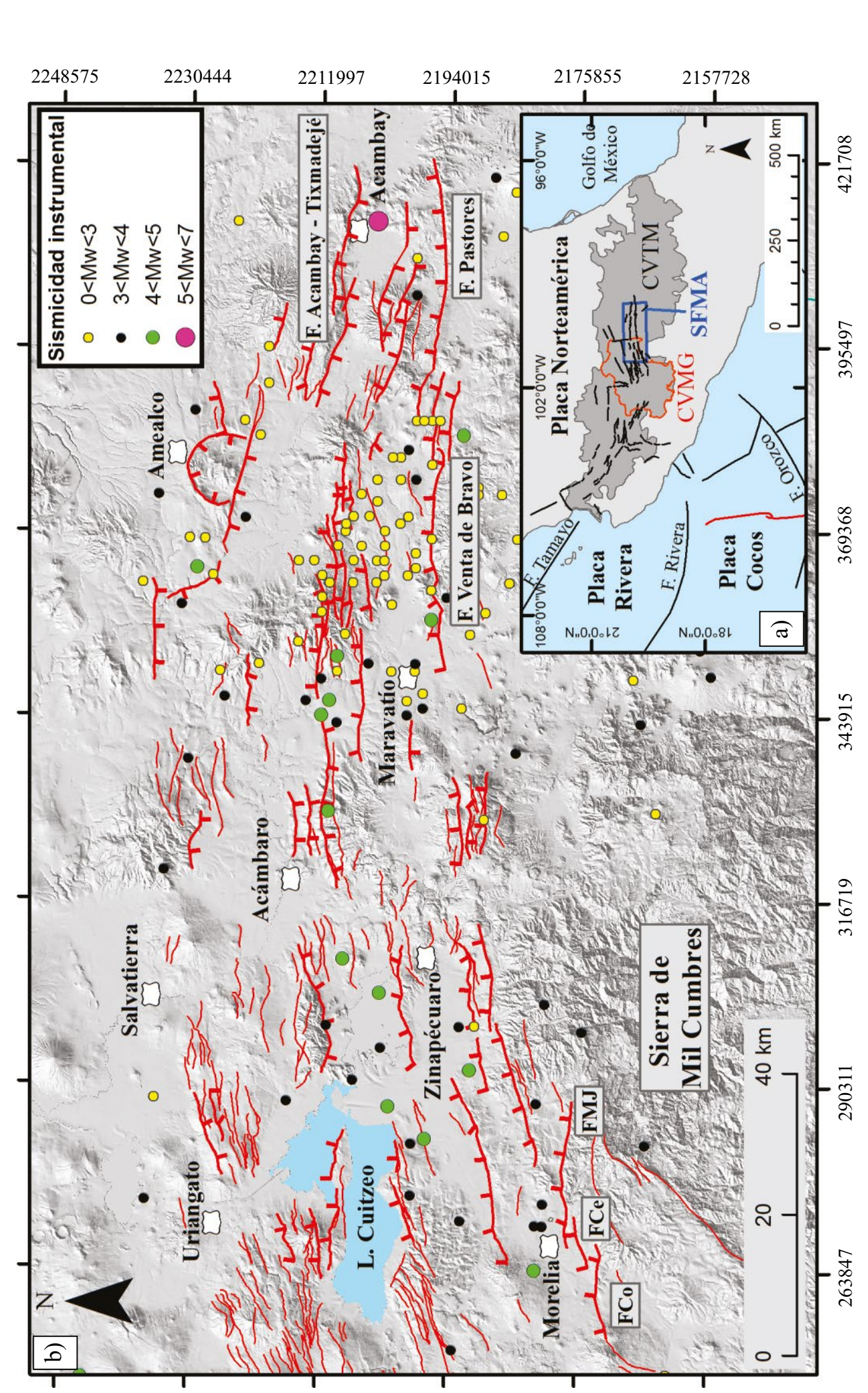

串

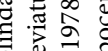

0

可

षु :

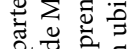

票要

ปี $\frac{3}{3}$ : 8

ङ

$=\frac{1}{2}$

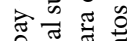

ही

प

吼

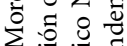

o

ज.

क क

뭉

政

is

$\bar{\nabla} \approx$

흠

든 쟁

일

ज

응

ज造。

记

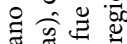

可

漹

守进

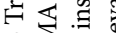

$8 \sum^{2}$

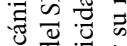

讨

듬. 워

表范

웜

요의

䒕全苛

언

낭

证

O욜

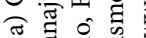

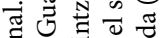

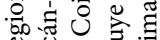

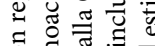

은 둔

赵就递

응 की

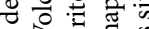

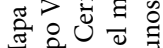

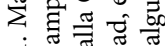

管部离高 


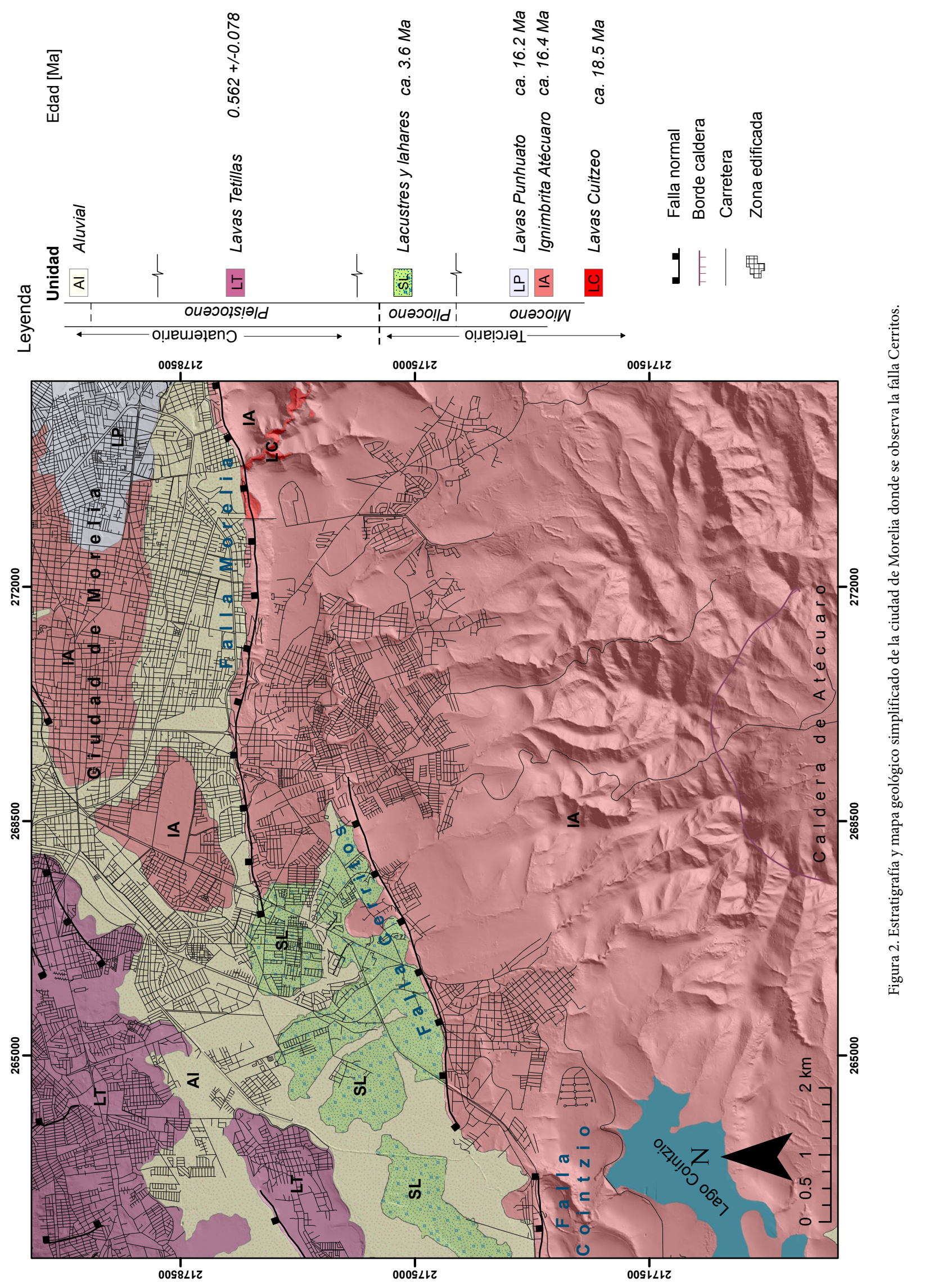


de Atécuaro por depósitos del vulcanismo monogenético asociado al campo volcánico Michoacán-Guanajuato, y en la zona de estudio por sedimentos fluvio-lacustres y depósitos aluviales (Gómez-Vasconcelos et al., 2015; Avellán et al., 2020).

El CVTM representa una deformación tectónica principalmente extensional que se expresa en sistemas de fallas a lo largo y ancho de toda la provincia (Figura 1); aunque estas fallas son predominantemente normales, existe una componente de movimiento lateral izquierdo que puede relacionarse al movimiento del sur de México hacia el oriente debido al empuje de la subducción de la placa de Cocos por debajo de la placa Norteamericana, donde el CVTM actúa como una zona de debilidad (Suter et al., 2001; Suárez et al., 2019). Con base en la información macrosísmica recopilada para los últimos 500 años, se considera que el CVTM es sísmicamente activo en su totalidad (Chico Hernández y Ruiz Barón, 2017; Suárez et al., 2019), ya que los registros de sismicidad instrumental sólo comprenden los últimos 100 años, con tres eventos sobresalientes (sismo de Maravatío en 1979, Ms 5.6, sismo de Acambay en 1912, $M_{\mathrm{W}} 7.0$ y sismo de Jalapa en 1920, $M_{\mathrm{W}} 6.4$ ).

En el sector central del CVTM se localiza el SFMA, el cual presenta evidencias de actividad continua y recurrente, que ha justificado los estudios enfocados en la potencialidad sísmica de las fallas que lo componen y su peligrosidad asociada.

\section{Sistema de Fallas Morelia-Acambay}

El SFMA abarca un conjunto de fallas en su mayoría normales con geometrías segmentadas y orientaciones E-O, ENE-OSO y NE$\mathrm{SO}$, distribuidas en un área de $\sim 60 \mathrm{~km}$ de ancho y $200 \mathrm{~km}$ de largo, atravesando la porción noreste de Michoacán, parte del estado de Guanajuato y del Estado de México. Este sistema se originó durante el Mioceno temprano con movimientos laterales izquierdos a transtensivos, y entre 12-6 Ma cambió a movimientos extensionales (Ferrari et al., 1990; Garduño-Monroy et al., 2009a; Mennella, 2011; MendozaPonce et al., 2018).

El SFMA presenta el desarrollo de grabens y semi-grabens de dimensiones variables. Los mecanismos focales de la región muestran la presencia de las fallas hasta la base de la corteza sismogenética ( 15 km; Rodríguez-Pérez y Zúñiga, 2017) que superficialmente afectan litologías con edades desde el Mioceno hasta suelos recientes. El sector oriental del sistema incluye el graben de Acambay, así como las fallas con mayor longitud ( $>32 \mathrm{~km}$ ) y con escarpes relativamente altos (>200 m) (p.ej., fallas Pastores y Venta de Bravo; Ortuño et al., 2015); además, cuenta con una buena cantidad de información sobre la actividad sísmica histórica e instrumental. El sector occidental abarca las cuencas del lago de Cuitzeo y el horst de Santa Ana Maya; este sector presenta fallas con longitudes mayores a $10 \mathrm{~km}$ que influyen en la distribución de centros eruptivos holocénicos (Garduño-Monroy et al., 2009a; Israde-Alcántara et al., 2010; Suter, 2016; Avellán et al., 2020; Gómez-Vasconcelos et al., 2020). Este sector posee menos información sobre la actividad de las fallas.

La información macrosísmica disponible para el SFMA incluye registros de crisis de sismicidad en los años 1734 a 1755 y 1853 a 1854, cerca de la falla Venta de Bravo (Suter et al., 1992), sismos pequeños en el graben de Mezquital (Quintanar et al., 2004), los sismos de Araró del 15 de mayo de 1845 (Cortés-Arreola, 1985), el enjambre sísmico en la región de Ucareo-Zinapécuaro-Acámbaro en octubre de 1872 y noviembre de 1874 (Orozco y Berra, 1887; Urquiza, 1872; Ramírez y Reyes, 1873, contenidas en Cortés-Arreola, 1985; Dobson y Mahood, 1985), así como los registros de sismicidad en la cuenca de Pátzcuaro en los años 1603, 1758, 1801, 1837, 1845 y 1895, siendo el de mayor intensidad el sismo de 1845 (VIII en la escala de Mercalli; SánchezGarcilazo, 2000; Garduño-Monroy et al., 2009b), esto sin considerar el sismo del 19 de junio de 1858 de intensidad VIII-IX en la escala de
Mercalli modificada, el cual se describe como un evento intraplaca ligado a la subducción (Singh et al., 1996).

Respecto a la sismicidad instrumental, los registros del Servicio Sismológico Nacional muestran una actividad recurrente de sismos de magnitudes bajas y moderadas distribuidos a lo largo de todo el SFMA (Figura 1), pero los eventos más significativos han sido: el sismo de Acambay, el 19 de noviembre de 1912, el cual tuvo una magnitud de $M_{\mathrm{W}} 7.0$ (Urbina y Camacho 1913; Abe, 1981; Langridge et al., 2000) y ocasionó al menos un centenar de decesos, al igual que cuantiosos daños en la capital del país (Suter, 2014) y el sismo de Maravatío en 1979, que tuvo una magnitud de Ms 5.6 y un hipocentro a $8.2 \mathrm{~km}$ (Astíz-Delgado, 1980; Garduño-Monroy y Gutiérrez-Negrín, 1992). En el 2007, ocurrieron siete sismos en Morelia relacionados a la falla Morelia, tres de ellos con magnitudes de $M_{\mathrm{W}} 4.3$ (Garduño et al., 2007; Singh et al., 2012; Suter, 2016).

Respecto a la potencialidad sísmica de las fallas del SFMA, los análisis paleosísmicos de algunas de las fallas principales han arrojado resultados importantes. Se han estimado tasas de desplazamiento vertical de $0.1 \mathrm{~mm} /$ año y tasas de extensión de $0.2 \pm 0.05 \mathrm{~mm} /$ año (Langridge et al., 2000; Suter et al., 2001), con variaciones entre sus sectores. Para el sector oriental se han estimado tasas de desplazamiento promedio de 0.03 a $0.37 \mathrm{~mm} /$ año (Suter et al., 1992; Langridge et al., 2000; Ortuño et al., 2015), con tiempos de recurrencia que van de 1100 a 11570 años (Ortuño et al., 2015; Sunye-Puchol et al., 2015) y magnitudes potenciales de $M_{\mathrm{W}} 6.4$ a 7.0 (Langridge et al., 2000; Ortuño et al., 2015 Sunye-Puchol et al., 2015; Arzate et al., 2018; Lacan et al., 2018; Ortuño et al., 2019). Mientras que en el sector occidental se han estimado tasas de desplazamiento promedio de 0.01 a $2.78 \mathrm{~mm} /$ año, con tiempos de recurrencia que van de 1200 a 20000 años y magnitudes potenciales de $M_{\mathrm{W}} 5.8$ a 7.2 (Garduño-Monroy et al., 2009a; Suter, 2016; Soria-Caballero et al., 2019; Gómez-Vasconcelos et al., 2021).

En la porción occidental del SFMA se ubica la falla Cerritos (Garduño-Monroy et al., 2001; Suter, 2016), también conocida como falla Morelos (Garduño-Monroy et al., 2009a), para la cual se han calculado tasas de desplazamiento de 0.009 a $0.03 \mathrm{~mm}$ /año (GarduñoMonroy et al., 2009a y Gómez-Vasconcelos et al., 2021, respectivamente), tiempos de recurrencia de 16900 años a 100000 años (corto y largo plazo) y magnitudes potenciales de $M_{\mathrm{W}} 5.9$ a 6.6 (Garduño-Monroy et al., 2009a; Gómez-Vasconcelos et al., 2021), por lo que se ha considerado como una falla potencialmente activa, cuyo estudio es relevante para el peligro sísmico de la ciudad de Morelia y el estado de Michoacán.

\section{MÉTODOS}

El análisis de la falla Cerritos se llevó a cabo en dos aproximaciones: una caracterización geológica-geomorfológica y una caracterización geofísica.

Para la caracterización geológica se utilizaron como insumos la carta topográfica escala 1:50000 del municipio de Morelia, imágenes satelitales (SPOT 5, pancromática con tamaño de píxel de $2.5 \mathrm{~m}$ y multiespectral $10 \mathrm{~m}$, ERMEXS) y ortofotos ( 2.5 y $1 \mathrm{~m}$; escala $1: 10000$ y 1:20000), obtenidas del Instituto Nacional de Estadística, Geografía e Informática (INEGI) y la Secretaría de Marina (SEMAR), con resolución de $15 \mathrm{~m}$, así como estudios previos en la región (p.ej., Gómez-Vasconcelos et al., 2015; Avellán et al., 2020). Con esta información se construyó una cartografía geológica que fue integrada a un modelo digital topográfico sombreado del terreno (DEM, por sus siglas en inglés: Digital Elevation Model), proyectado en el sistema de coordenadas WGS-1984-UTM-Zona-14N, con el cual también se construyeron mapas temáticos de disección vertical y pendientes, usando la plataforma del programa informático ArcMap 10.2. Con la 
cartografía geológica se identificaron las unidades litoestratigráficas afectadas por la zona de falla Cerritos y se realizó una reconstrucción simplificada de la estratigrafía de la zona, mientras que con la cartografía temática se obtuvieron los rasgos morfométricos más importantes (Tabla 1 y Tabla S1 del suplemento electrónico) vinculados a la zona de falla. En el trabajo de campo se verificaron los contactos litológicos trazados, el levantamiento estratigráfico, se validaron las trazas de falla digitalizadas para su análisis estructural, se realizó la medición de los planos de falla para caracterizar su geometría y calcular los tensores de esfuerzos. Los indicadores cinemáticos utilizados para el cálculo del campo de esfuerzos fueron estrías, estructuras de Riedel y fracturas extensivas sin-tectónicas.

Para el análisis de índices geomórficos se calcularon parámetros de cuencas de drenaje como son: el parámetro $K f$ de Horton (1932), la curva hipsométrica e integral hipsométrica $(I H)$, el factor de asimetría $(F A)$, el cual se obtiene a partir del trazado de la configuración de cada cuenca y cuya clasificación se basó en los estudios de Özkaymak y Sözbilir (2012) y Tepe y Sözbilir (2017). Asimismo, se usó la relación ancho-altura del valle $(V f)$ y la sinuosidad del frente de montaña $(S f m)$ para analizar la geometría de la zona del escarpe; esto requirió del trazado de perfiles de valles transversales (para determinar $V f$ ) a $c a$. $0.6 \mathrm{~km}$ aguas arriba del frente de montaña para las cuencas identificadas (ver material suplementario).

Tabla 1. Índices geomórficos de las cuencas de drenaje del frente de montaña asociado a la zona de falla Cerritos.

\begin{tabular}{lcccccl}
\hline Cuenca & $\begin{array}{c}\text { Área } \\
\left(\mathrm{km}^{2}\right)\end{array}$ & $\begin{array}{c}\text { Longitud } \\
\left(\mathrm{km}^{2}\right)\end{array}$ & ${ }^{\star} \boldsymbol{K} \boldsymbol{f}$ & ${ }^{\mathrm{I}} \boldsymbol{I H}$ & ${ }^{\mathrm{F}} \boldsymbol{F} \boldsymbol{~}$ & $\boldsymbol{}_{\boldsymbol{V} \boldsymbol{f}}$ \\
\hline $\mathrm{C} 1$ & 3.4 & 3.6 & 0.3 & 0.6 & 43 & 0.7 \\
$\mathrm{C} 2$ & 6.7 & 5.5 & 0.2 & 0.7 & 75 & 1 \\
$\mathrm{C} 3$ & 0.8 & 2 & 0.2 & 0.5 & 59 & 2 \\
$\mathrm{C} 4$ & 3.4 & 4.1 & 0.2 & 0.6 & 67 & 0.8 \\
$\mathrm{C} 5$ & 9.7 & 5.6 & 0.3 & 0.6 & 75 & 0.2 \\
$\mathrm{C} 6$ & 1.7 & 3.1 & 0.2 & 0.6 & 57 & 0 \\
C7 & 18.9 & 7.1 & 0.4 & 0.5 & 83 & 0.0 \\
\hline
\end{tabular}

Nota: Parámetros de cuencas de drenaje establecidos por Horton (1932), Özkaymak y Sözbilir (2012), y Tepe y Sözbilir (2017). Ver localización de las cuencas de drenaje en la Figura 6. ${ }^{*} \mathrm{Kf}$ : parámetro de cuenca de drenaje (Horton, 1932). ${ }^{\vee} I H$ : curva hipsométrica e integral hipsométrica. ' $F A$ : factor de asimetría. $~ V f$ : relación alto-ancho del valle. Ver Tabla Suplementaria 2.

La potencialidad sísmica de la falla (en magnitud momento $M_{\mathrm{W}}$ ) se estimó basándose en la longitud de ruptura superficial (LRS) y aplicando las relaciones empíricas de Wells y Coppersmith (1994) $\left(M_{\mathrm{W}}=4.80+1.33 \cdot \operatorname{logLRS}\right.$, Ecuación 1) y Wesnousky $(2008)\left(M_{\mathrm{W}}=\right.$ $6.12+0.47 \cdot \operatorname{logLRS}$, Ecuación 2). La tasa de desplazamiento se calculó a partir del desplazamiento vertical neto, considerando un ángulo de buzamiento promedio de $60^{\circ}$, a partir de los datos de campo y estudios regionales previos sobre la edad de los horizontes desplazados (ignimbrita Atécuaro; Garduño-Monroy et al., 2009a; Suter, 2016; Soria-Caballero et al., 2019; Avellán et al., 2020; Gómez-Vasconcelos et al., 2020). El período promedio de recurrencia (IR) se calculó usando la relación: $I R=D E / T M$ (Ecuación 3; Wallace, 1978), donde $D E$ es el desplazamiento de un sólo evento sísmico, y TM es la tasa de desplazamiento.

Adicionalmente, se analizó un afloramiento natural ubicado en el área de deformación del bloque levantado (ver localización en Figura 3). Los materiales aquí expuestos fueron identificados, descritos y analizados siguiendo la metodología paleosísmica tradicional: limpieza de la pared, registro fotográfico (fotolog) y log dibujado a una escala de 1:20.
En este afloramiento se tomó una muestra de sedimento orgánico para su fechamiento por radiocarbono en el laboratorio de Beta Analytic de Florida, EUA, usando espectrometría de masas con aceleradores NEC y IRMSsThermo. La edad de radiocarbono convencional se calculó utilizando la vida media establecida por Libby (1961; 5568 años). La edad convencional fue calibrada usado INTCAL20. Los resultados están acreditados por la normativa ISO/IEC-17025:2017 (Ramsey, 2009; Reimer et al., 2020).

Con el objetivo de conocer la geometría de la falla Cerritos en profundidad, se realizaron estudios de sísmica de refracción, tomografía de resistividad eléctrica y magnetometría terrestre sobre perfiles con orientaciones transversales a la dirección de la traza de falla principal (Figura 3).

En el caso de la refracción sísmica se realizaron tres perfiles ( $\mathrm{S} 1$, S2 y S3), cada uno de $120 \mathrm{~m}$ de longitud, dispuestos en solape para aumentar la cobertura en las zonas de interés y mejorar la resolución en puntos críticos. Los perfiles S1 y S2 se solapan $55 \mathrm{~m}$ en el extremo norte del perfil S1 y los perfiles S2 y S3 se solapan $30 \mathrm{~m}$ en el extremo norte del perfil S2. Con este arreglo se obtiene una mejor resolución de las interfases someras del subsuelo y permite determinar las profundidades de las mismas con mayor precisión (Figura 3c). Las secciones sísmicas resultantes se integraron en un sólo perfil de $260 \mathrm{~m}$ de longitud (ST; Figura 3c) para la elaboración del modelo final de velocidades. Para la adquisición de datos se usó un sismómetro modelo GEODE de 24 canales (marca GEOMETRICS) con 24 geófonos de $4.5 \mathrm{~Hz}$ espaciados cada $5 \mathrm{~m}$ y un marro de $8 \mathrm{~kg}$ como fuente generadora de ondas acústicas. Los puntos de disparo fueron colocados en los extremos y en el centro de cada perfil (ver Figura 3c). Cada sección sísmica tuvo un tiempo máximo de registro de $0.5 \mathrm{~s}$, y todos los registros sísmicos fueron adquiridos con una tasa de muestreo de 4000 muestras/s. El procesamiento de datos se realizó con el programa informático SeisImager $/ 2 D^{\mathrm{TM}}$ y sus dos módulos: Pickwin ${ }^{\mathrm{TM}}$ v. 5.1.1.2 para marcar las primeras llegadas de la onda sísmica, y Plotrefa $^{\mathrm{TM}}$ v. 3.0.0.6 para el modelado e interpretación. Para cada punto de disparo se generó una sección sísmica y se identificaron los primeros arribos en cada una de las trazas de los perfiles S1, S2 y S3. Se analizaron las curvas distanciatiempo de viaje del perfil ST y se asignaron tres capas (rojo=capa 1; verde $=$ capa 2 ; azul=capa 3 ) con sus respectivas velocidades (Figura $3 \mathrm{~d}$ ). Posteriormente, se aplicó el método de inversión de tiempo y se generó el modelo de las variaciones de velocidad de ondas P para el perfil ST.

Para la Tomografía de Resistividad Eléctrica se realizó una línea de adquisición de datos de $1025 \mathrm{~m}$ de longitud, utilizando un resistivímetro SuperSting R1 de 8 canales, un switch box y siete cables pasivos marca AGI, así como 42 electrodos y una batería de ciclo profundo para proveer de energía eléctrica al resistivímetro. Para este estudio se usó una apertura entre electrodos de $25 \mathrm{~m}$ y se propuso un arreglo DipoloDipolo (Figura S1del suplemento electrónico), debido a la sensibilidad que tiene a cambios horizontales en la resistividad, siendo útil para localizar estructuras predominantemente verticales como fallas y fracturas (Loke, 2015). Las características generales de la instalación de la línea se incluyen en el suplemento electrónico (Tabla S2). Los parámetros de adquisición fueron programados con el programa informático $A G I$ SuperSting Administrator, donde se programaron sub-arreglos Max. "a" para incrementar el espaciamiento entre los dipolos cuatro veces, con el objetivo de incrementar la profundidad de investigación hasta 180 metros teóricos. Estos datos fueron procesados usando el programa informático de inversión AGI EarthImager ${ }^{\mathrm{TM}} 2 \mathrm{D}$ para generar un archivo TRN, considerando los parámetros de número de iteraciones a realizar, el tipo de datos de entrada o modelo inicial, factores de suavizado, y las condiciones de paro del programa basadas en la convergencia de cada iteración y la disminución del error entre la iteración actual y la anterior.

Para la magnetometría terrestre se adquirieron datos a lo largo de 

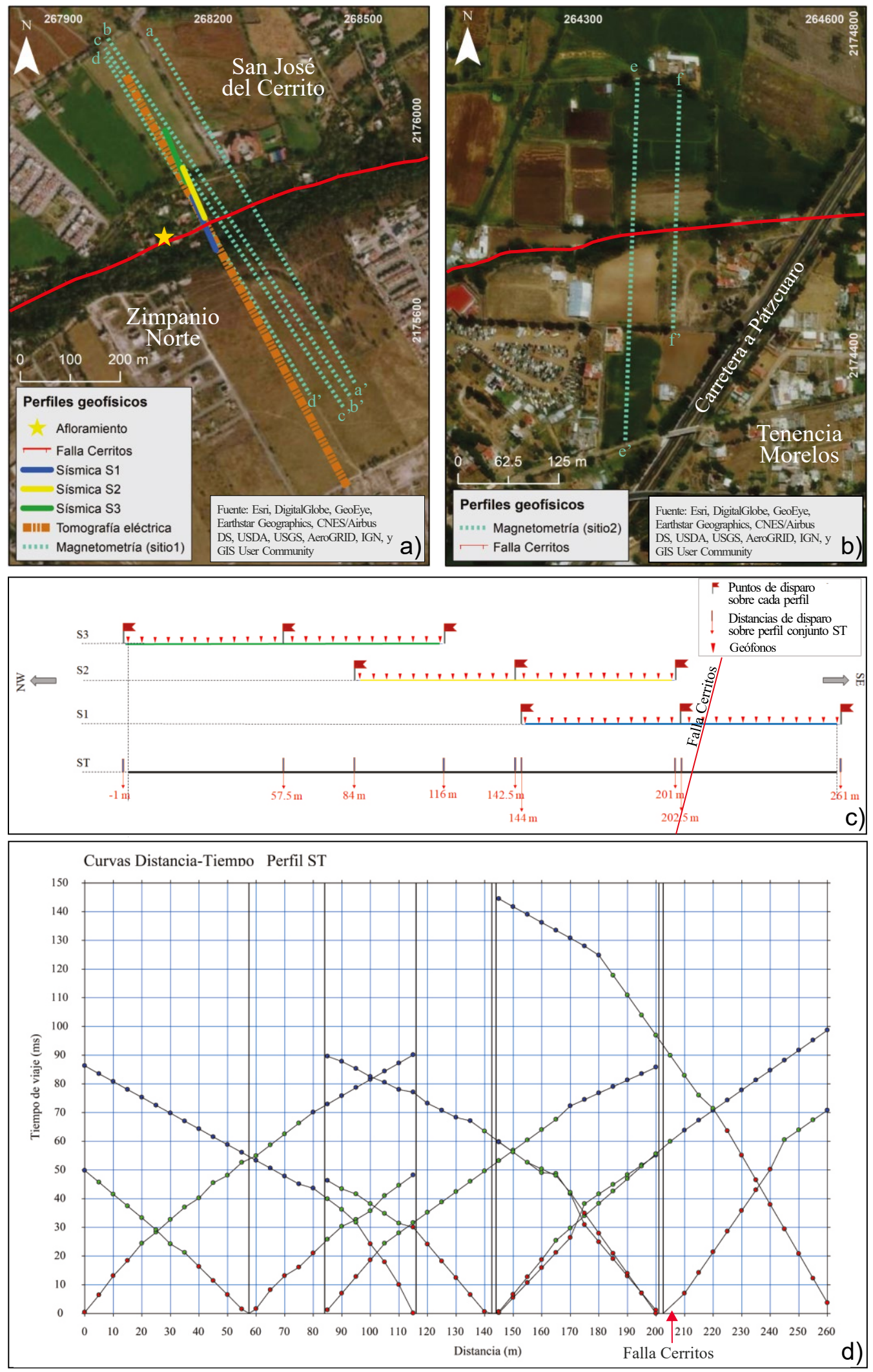

Figura 3. a) Arreglo de los perfiles geofísicos en el sitio 1. b) Arreglo de los perfiles de magnetometría en el sitio 2 (ver localización de los sitios en la Figura 5). c) Diagrama de la metodología empleada para el estudio de sísmica de refracción, donde se muestra la disposición de los perfiles de adquisición, indicando la ubicación de cada fuente de disparo y el arreglo de los receptores. d) Curvas distancia-tiempo para el perfil sísmico conjunto, indicando la asignación de las capas de velocidad para el modelo. Rojo corresponde a la capa más somera, verde la capa intermedia y azul la capa más profunda. 
seis perfiles: cuatro en la porción oriental de la falla Cerritos (sitio 1) y dos en la porción occidental (sitio 2; Figura 3). Los datos se adquirieron en tres campañas, empleando para las estaciones móviles dos magnetómetros de precesión de protones G-857 de la marca Geometrics con resolución de $0.1 \mathrm{nT}$, y para la base un magnetómetro de efecto Overhausser GSM-19 de GEM-Systems con resolución de 0.01 nT. Para la porción oriental se tomaron datos cada $20 \mathrm{~m}$, resultando en un largo de 850 metros; mientras que para la porción occidental la separación entre estaciones fue de 15 metros, resultando en perfiles de 350 y $450 \mathrm{~m}$ de longitud. Los datos fueron corregidos por el campo geomagnético terrestre y variación diurna, usando la información de la estación base para la porción oriental y los datos del Observatorio Geomagnético de Teoloyucan para la porción occidental, proporcionados por el Dr. Esteban Hernández. Se utilizó el programa informático Oasis Montaj (Geosoft) obteniendo el mapa de campo geomagnético residual, al cual se aplicó el filtro de reducción al Polo (CMRP) con el objetivo de centrar las anomalías magnéticas sobre los cuerpos que las generan. A partir del mapa CMRP, se aplicaron los filtros de gradiente horizontal y pasa-bajas, con el fin de realizar un análisis comparativo de las anomalías obtenidas a lo largo del perfil topográfico y su correlación con la falla Cerritos.

\section{RESULTADOS}

\section{Geología y Geomorfología}

Estratigrafía en la zona de falla Cerritos

En la zona de estudio, la falla Cerritos afecta a la ignimbrita Atécuaro de 16.4 Ma (Figura 2) (Gómez-Vasconcelos et al., 2015). Por las características identificadas a lo largo del escarpe de la falla Cerritos, la ignimbrita Atécuaro se reconoce como un depósito de corriente de densidad piroclástica concentrada muy bien soldada, Figura 4. Se identificó una porción inferior con estructura en entabladura (altamente diaclasada) y una porción superior con estructura masiva a columnar difusa, con un bajo contenido de fragmentos de pómez ( 5 vol.\%) de hasta $1.5 \mathrm{~cm}$ de color blanco, soportado por una matriz de coloración gris-rosáceo claro a gris muy claro de textura porfirítica y fenocristales de plagioclasa, cuarzo, anfíboles y sanidino.

En el bloque caído, la ignimbrita Atécuaro se encuentra sobreyacida por una secuencia de sedimentos lacustres y flujo de detritos (Figura 2). En las diferentes columnas estratigráficas realizadas en campo, es común ver aflorar al menos tres capas de sedimentos lacustres

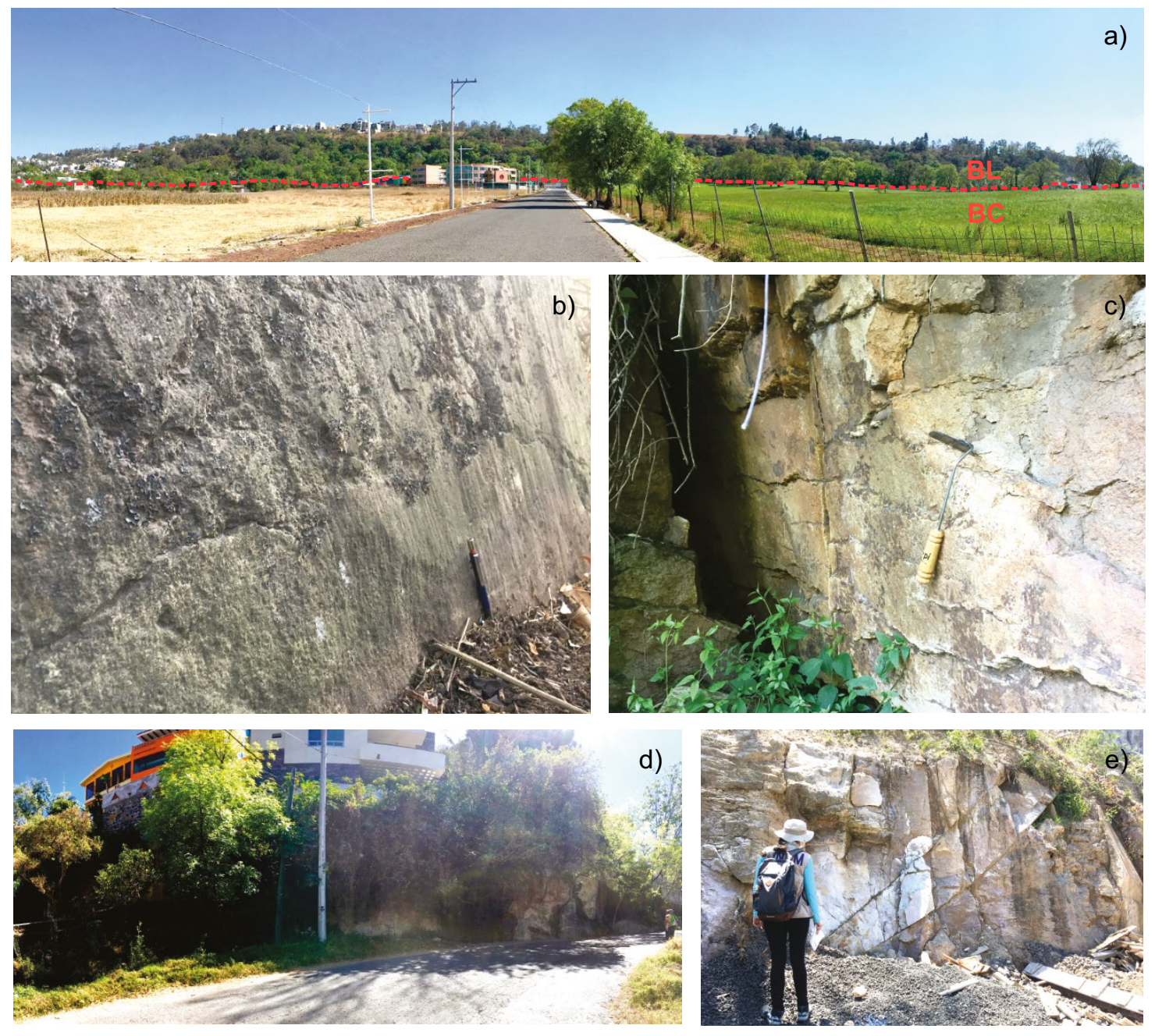

Figura 4. Fotografías de campo. a) Escarpe principal de la falla Cerritos visto de Norte a Sur, que corresponde al sitio 1 donde se realizaron los estudios geofísicos (colonia San José del Cerrito, al Sur de Morelia). BL, Bloque Levantado; BC, Bloque Caído. b) Detalle de estrías en el afloramiento del escarpe de falla (estación estructural 1) conformado por la ignimbrita Atécuaro y depósitos superficiales. Esta falla normal tiene un rumbo de $281^{\circ}$, buzamiento de $71^{\circ}$ al NNE, y pitch de $88^{\circ}$. c) Fractura extensiva tectónica localizada en la estación estructural 2 (Figura 5). d) Escarpe de la falla Cerritos en la estación estructural 3 (subida a Zimpanio Norte). e) Afloramiento de la ignimbrita Atécuaro fallada y fracturada (estación estructural 5). 
consecutivamente estratificadas en contacto directo y erosivo, las cuales presentan una estructura masiva y endurecida. En la porción inferior es común encontrar lentes de grava y arena muy redondeada; la capa intermedia se compone por arcillas de color gris claro que desarrollan grietas de desecación; en la cima de la secuencia se observa una capa enriquecida en arcillas blancas con estructura laminar, las que posiblemente correspondan a facies enriquecidas en diatomeas. En el área de estudio, se desconoce la edad de estas capas lacustres y lahares interestratificados, sin embargo, se podrían correlacionar con los depósitos lacustres reportados por Carranza-Castañeda (1976) al oriente de Morelia con una edad de 3.6 Ma (en el bloque caído de las fallas Charo y Morelia).

\section{Geometría y datos de la falla Cerritos}

La zona de falla Cerritos consiste en un arreglo en échelon de trazas de falla con una longitud máxima de $12 \mathrm{~km}$, una dirección ENE-OSO y un buzamiento NNO. En la zona de falla resalta la presencia de un segmento principal que muestra el mayor desplazamiento y actividad (Figuras 4 y 5b), aquí referido como falla Cerritos, en el cual se enfocó este trabajo.
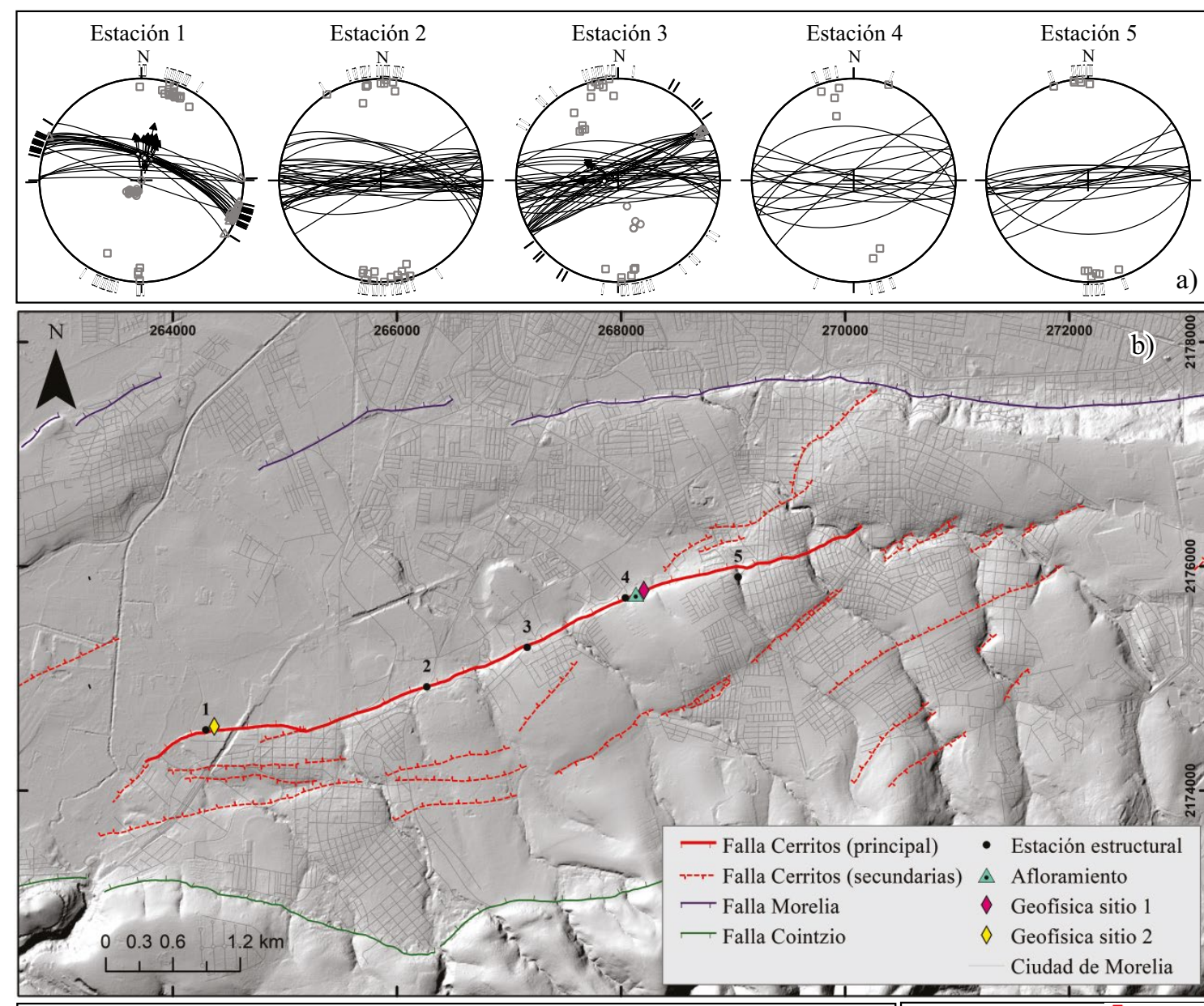

\begin{tabular}{lr}
$1 . \ldots$ \\
\hline 1000 & 272000
\end{tabular}

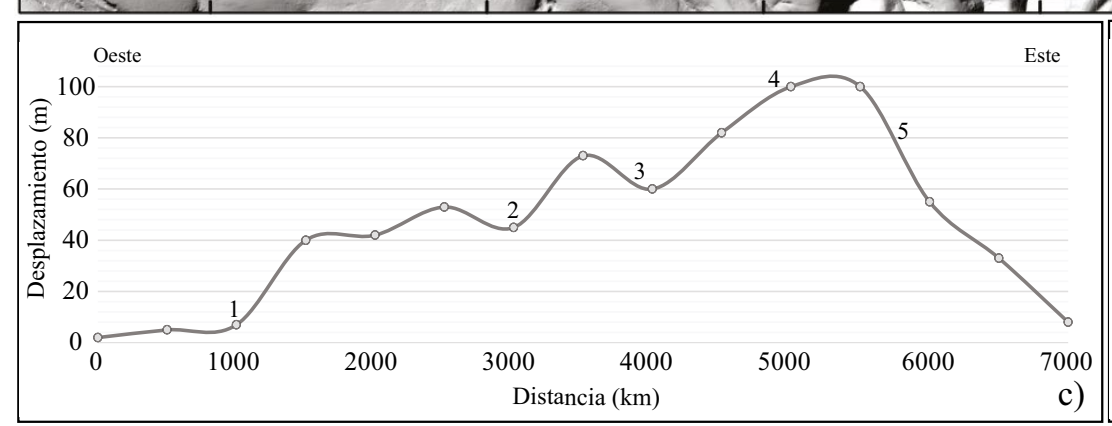

-


000
c)

b) $\frac{5}{\frac{0}{2}}$

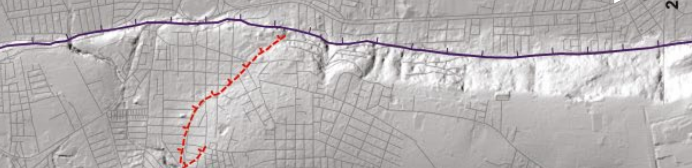


La falla Cerritos es una falla normal lístrica de dirección ENE-OSO (rumbo entre $230^{\circ}$ y $278^{\circ}$, con un valor medio de $255^{\circ}$ ), con el bloque caído buzando al NNO (ángulo de inclinación con un valor promedio de $76^{\circ}$ ) y basculamiento en el bloque levantado variable entre $8^{\circ}$ y $22^{\circ}$ al SSE. Este segmento posee una longitud de $7 \mathrm{~km}$ y un escarpe superficial bien definido que presenta una distribución asimétrica del desplazamiento a lo largo de la falla, con un escarpe vertical visible máximo de $100 \mathrm{~m}$ en su parte central-oriental y mínimo de $4 \mathrm{~m}$ en su parte occidental (Figura 5 b y $5 c$ ), desplazando a la ignimbrita Atécuaro.

Las fallas y fracturas medidas se pueden agrupar en tres familias principales: NNO-SSE, NE-SO y E-O (Figura 5a). La primera familia es la más antigua y está representada por estructuras NNO con movimiento de rumbo dextral; la segunda familia se caracteriza por estructuras con orientación NE con movimiento normal a sinestral. Mientras que la familia E-O suele ser la más reciente que corta a las demás familias; está representada por fracturas extensivas y fallas con movimiento normal y una ligera componente sinestral (Figuras 4 y 5 ).

\section{Índices geomórficos}

El área de estudio presenta un relieve de montaña bajo a moderado en la parte sur, altiplanicie ligeramente inclinada en la zona central y planicies en toda la zona norte. Las elevaciones máximas sobre el nivel del mar van desde $1900 \mathrm{~m}$ en las zonas más bajas al norte con pendientes entre $0^{\circ}$ y $3^{\circ}$, hasta $2580 \mathrm{~m}$ en las partes más altas al sur con pendientes entre $45^{\circ}$ y $85^{\circ}$. El cambio en el paisaje geomorfológico integra el flanco norte de la caldera de Atécuaro y se considera el resultado de la interacción entre la tectónica activa regional y los procesos erosivos de superficie, propios del SFMA. La zona de falla Cerritos presenta características típicas de un frente de montaña (p.ej., Keller y Pinter, 1996) con marcadores geomórficos dados por un lineamiento de frente de montaña en la zona del escarpe que marca la frontera del bloque caído, y un sistema de ríos formados en el bloque levantado. En este trabajo se determinaron siete cuencas de drenaje (C1-C7 de oeste a este; Figura 6) para el bloque levantado, con geometría paralela y perpendiculares a la falla Cerritos, con áreas de superficie que van de 0.81 a $9.7 \mathrm{~km}^{2}$ (Tabla 1). La mayoría tienen una geometría muy alargada a ligeramente alargada, descrita cuantitativamente en la Tabla 1 usando el parámetro $K f$ (Horton, 1932) con valores calculados entre 0.19 y 0.37 .

Posterior al trazado de la línea divisoria, se calculó la curva hipsométrica e integral hipsométrica $(I H)$ para cada una de las cuencas que fluyen con dirección noroeste hacia el bloque caído (Figura S2). Este parámetro refleja la etapa de erosión a la que se encuentran las cuencas. La mayoría se comportan como curvas hipsométricas convexas, en un rango de valores $I H$ entre 0.52 y 0.67 (Tabla 1). A excepción de la cuenca $\mathrm{C} 3$ que se comporta ligeramente con una forma de $\mathrm{S}$, y un valor $I H$ de 0.5 .

Los valores del factor de asimetría $(F A)$ permitieron clasificar las cuencas en dos clases: cuencas asimétricas inclinadas hacia el suroeste con valores de $F A>55$ (entre 57 y 83, C2 a C7); y cuencas asimétricas inclinadas hacia el noreste con valores de $F A<45$ ( 43 para C1, Figura 6, Tabla 1). En consecuencia, el $57 \%$ son cuencas fuertemente asimétricas y el $43 \%$ son suavemente asimétricas.

Finalmente, la mayoría de los valores medidos de $V f$ (relación ancho-altura del valle; Figura 6) de los valles a lo largo del frente de montaña son $V f<1$, a excepción del valle C3 que es de 2.03 (Tabla 1). Sin embargo, los drenajes localizados más al noreste (C5-C7) tienen valores equivalentes o muy cercanos a 0 (C5), reflejando perfiles de valles en forma de $\mathrm{V}$ profundamente incisos. En el análisis de la geometría del frente de montaña se adquirió la sinuosidad del frente de montaña $(\mathrm{Sfm})$ con un valor de 1.06 para la zona de escarpe de la falla Cerritos que carece de facetas triangulares y posee pendientes que oscilan entre $18^{\circ}$ y $85^{\circ}$.

\section{Análisis del afloramiento Cerritos}

El afloramiento estudiado se trata de un corte de cerca de $5 \mathrm{~m}$ de longitud y una altura variable entre 2 y $3.5 \mathrm{~m}$, perteneciente al bloque levantado de la falla Cerritos, ubicado 30 metros al norte del escarpe principal, en las coordenadas 14 Q 0268087, 2175737, a 1950 m s.n.m. (Figuras 3a, 5 y 7). Presenta una orientación NNO-SSE $\left(163^{\circ}-155^{\circ}\right)$, sobre un terreno con pendiente de $12.5^{\circ}$. Los materiales expuestos se conforman de la base a la cima por las siguientes unidades:

1) Ignimbrita Atécuaro poco alterada, color blanco, se considera como la única unidad que muestra a la ignimbrita in situ (Figura 7f).

2) Depósito enriquecido en arcillas color café oscuro, con abundante materia orgánica. Presenta geometría acuñada y en su base se observan fragmentos removilizados, de 1 a $4 \mathrm{~cm}$ de diámetro, de la ignimbrita basal (Figura 7e). Esta unidad posee características que sugieren que corresponde a una cuña coluvial, aunque esta interpretación requiere más análisis de trincheras.

3) Depósito enriquecido en arenas y arcillas color blanquecino, con porosidad visible que rellena el bloque caído en el afloramiento. Posee características similares a las de los depósitos de lahares de la región, por lo que fue clasificado como uno de ellos (Figura 7d).

4) Depósito del horizonte mineral del suelo, caracterizado por la presencia de arcillas formadas por pedogénesis mezcladas con bloques de ignimbritas removilizados del escarpe principal. Estos bloques se observan alterados a muy alterados y con diámetros mayores a $15 \mathrm{~cm}$ (Figura $7 \mathrm{~b}-7 \mathrm{c}$ ).

5) En la cima de la secuencia, se observa el suelo actual. Se trata de un horizonte orgánico bien desarrollado, con un espesor de $25 \mathrm{~cm}$ (Figura 7a).

Toda la secuencia es afectada por fracturamiento y fallamiento paralelo a la traza principal de la falla Cerritos. Las fallas y fracturas se agrupan en una gran familia con dirección preferencial ENE-OSO, y una familia menos numerosa de estructuras con dirección NE-SO (estación 4 en Figura 5). La falla principal de este afloramiento causa un desplazamiento vertical de la ignimbrita basal de $84 \pm 18 \mathrm{~cm}$ (usando como marcador el contacto entre la ignimbrita basal y el depósito arcilloso identificado como probable cuña coluvial), repartido en cinco escalones que presentan desplazamientos parciales de entre 5 y $28 \mathrm{~cm}$, los cuales se forman por varias fallas asociadas, paralelas en sentido y desplazamiento (parte inferior derecha en la Figura 7). Este desplazamiento vertical puede estar subestimado por la naturaleza del afloramiento que impidió la observación a mayor profundidad de la ignimbrita desplazada. Dentro de estos escalones, las fallas provocan una ligera apertura horizontal por rotación de bloques, albergando un depósito arcilloso con abundante materia orgánica, que puede corresponder a una cuña coluvial poco desarrollada. En el depósito relacionado a la cuña coluvial (Figura 7e) se tomó una muestra del material orgánico y se fechó por el método de radiocarbono en 3.37-3.21 cal ka AP (Figura 7, Tabla 2).

\section{Geofísica}

\section{Magnetometría terrestre}

En el sector oriental (sitio 1 en las Figuras $3 a$ y $5 b$ ) se observa la presencia de un alto magnético en el norte del perfil con orientación NO-SE y con intensidades variables entre $-100 \mathrm{nT}$ y $-70 \mathrm{nT}$, seguida por un bajo magnético en dirección opuesta con valores entre - 200 y -160 nT (Figura 8b). Destaca la anomalía magnética negativa que se corresponde con el punto de inflexión de la pendiente, muy próxima a la falla (Figura S3). Se observa la presencia de pequeñas anomalías de aproximadamente - $100 \mathrm{nT}$ que presentan una orientación NO-SE. Los altos magnéticos ubicados en el bloque levantado hacia el Este del 

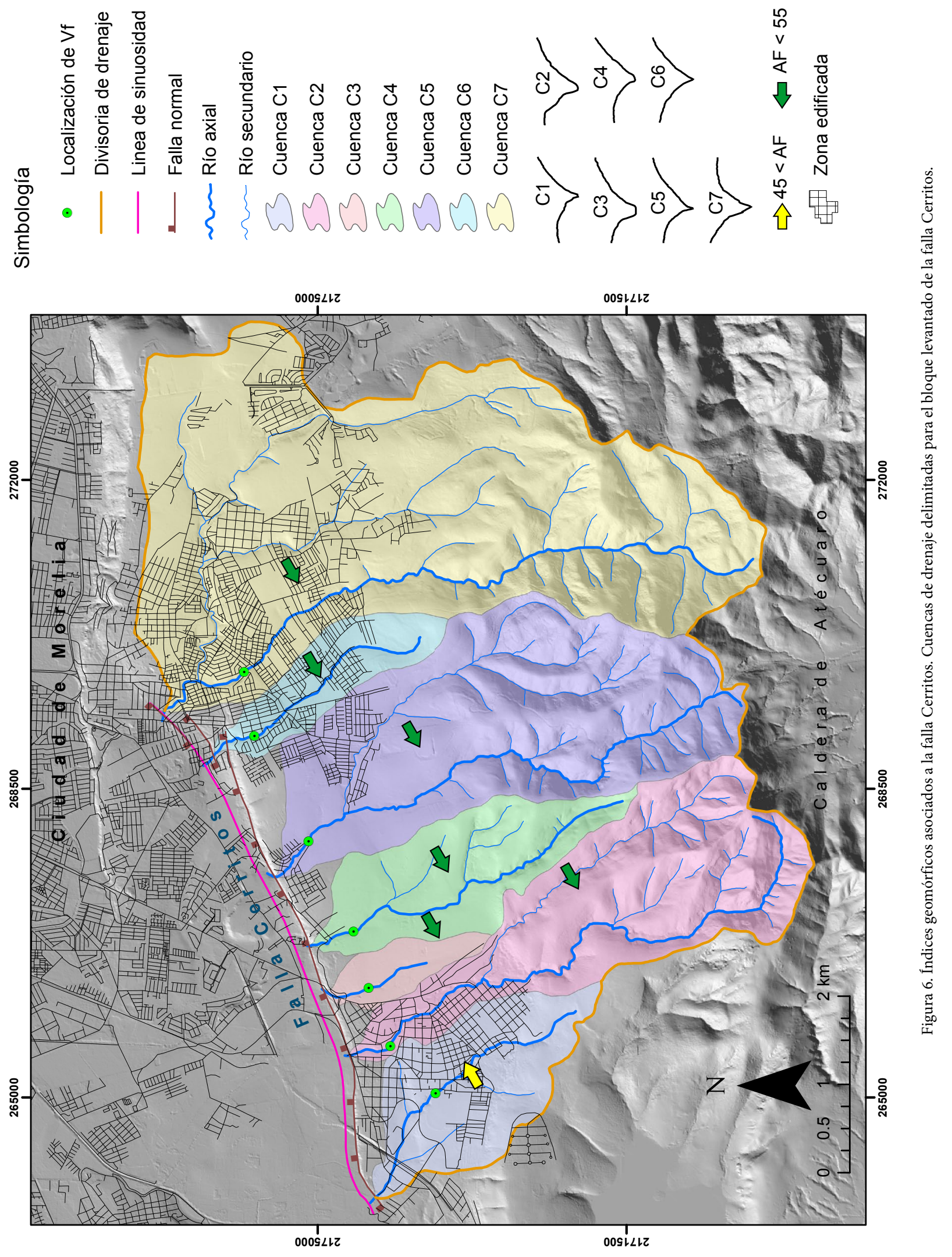

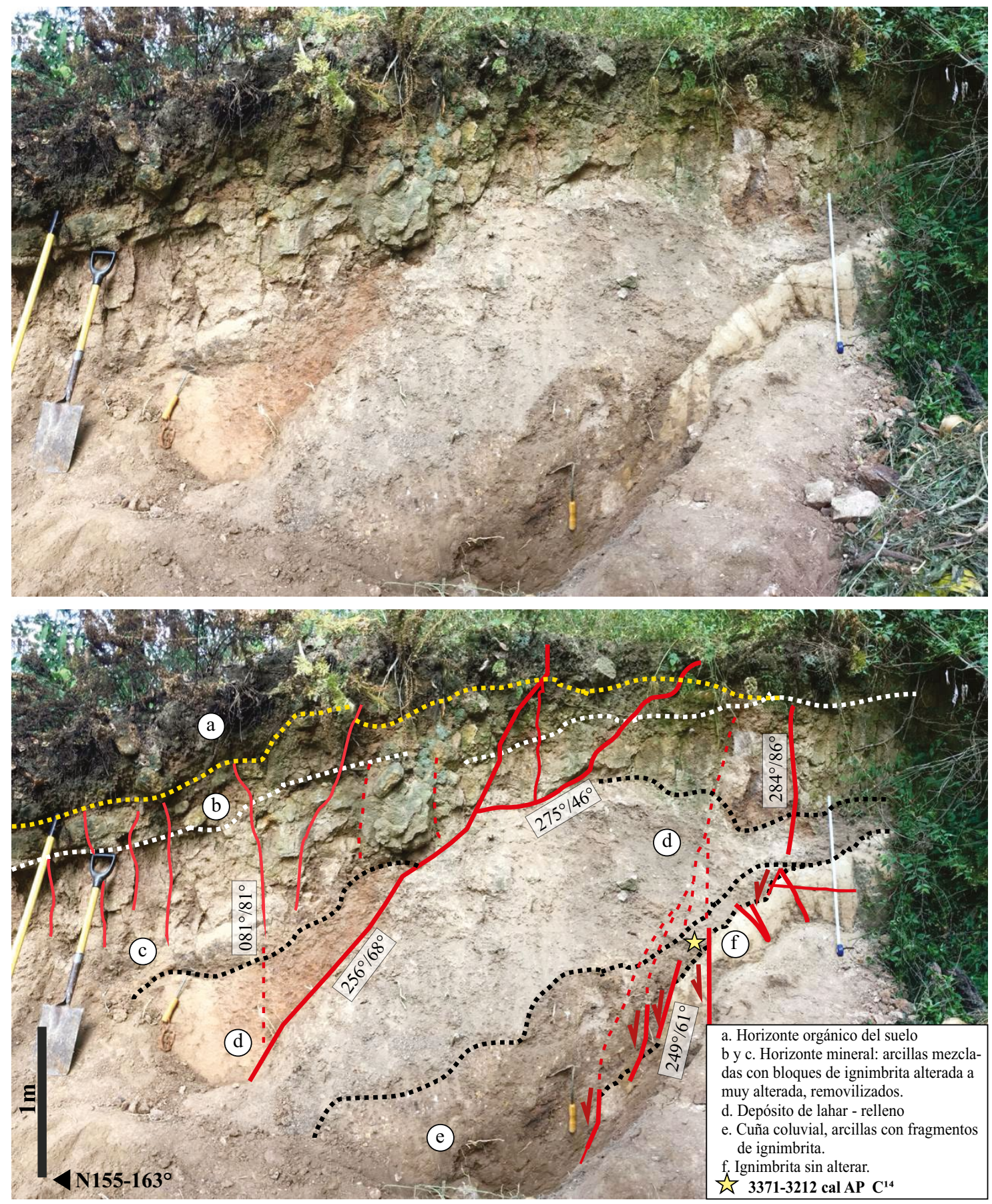

Figura 7. Composición fotográfica del afloramiento descrito en el bloque levantado de la falla Cerritos y fotolog interpretativo resaltando las unidades litológicas aflorantes, las fallas y fracturas principales (líneas rojas continuas), así como las fallas inferidas (líneas rojas discontinuas). Ver la ubicación del afloramiento en las Figuras $3 a$ y $5 b$.

mapa podrían estar asociados a artefactos generados por los parámetros de interpolación empleados (Figura 8b).

En el sector occidental (sitio 2 en las Figuras $3 b$ y $5 b$ ) se observa la presencia de altos magnéticos al norte de la falla con orientación NE-SO (Figura 8a), con intensidades entre $-100 \mathrm{nT}$ y $-80 \mathrm{nT}$ y que se corresponden con el bloque caído, mientras que los valores mínimos se concentran en el sur (bloque levantado).

La Figura S3 muestra una comparación de los filtros aplicados (campo magnético residual reducido al polo, gradiente horizontal y filtro pasa-bajas) a lo largo de su correspondiente perfil topográfico. Para el sector oriental de la falla (representado por el perfil C-C, Figura 3a), se observa la coincidencia del campo magnético residual con las anomalías resultantes del gradiente horizontal y filtro pasa-bajas, mostrando un comportamiento similar de las curvas graficadas, lo cual se corresponde con la presencia de la falla Cerritos en superficie (Figura S3). En cambio, el sector occidental de la falla, representado por el perfil F-F', muestra más contrastes en las anomalías resultantes; aquí se observa la coincidencia del comportamiento de las curvas con 
Tabla 2. Fechamiento por radiocarbono de la falla Cerritos.

\begin{tabular}{|c|c|c|c|c|c|c|}
\hline Muestra & $\begin{array}{l}\text { Número de } \\
\text { Laboratorio }\end{array}$ & $\begin{array}{c}\text { Edad }{ }^{14} \mathrm{C} \\
\text { convencional (AP) }\end{array}$ & $\begin{array}{c}\text { Edad }{ }^{14} \mathrm{C} \\
\text { calibrada (cal AP) }\end{array}$ & $\begin{array}{c}\text { Edad }{ }^{14} \mathrm{C} \\
\text { calibrada (cal a.C.) }\end{array}$ & $\begin{array}{l}\text { Relación de isótopos } \\
\text { estables }\left(\delta^{13} \mathrm{C}\right)\end{array}$ & Material analizado \\
\hline 1 Falla Cerritos & Beta - 583157 & $3080 \pm 30$ & $3371-3212$ & $1422-1263$ & -22.4 & sedimento orgánico \\
\hline
\end{tabular}

Nota: Edad obtenida por el método de radiocarbono por espectrometría de masas con aceleradores (AMS, ver Métodos). Edad más probable en negritas (95.4\%). Los valores $\delta 13 \mathrm{C}$ corresponden al material mismo (no al valor $\delta^{13} \mathrm{C}$ obtenido por AMS). AP, Antes del Presente; a.C., antes de Cristo.

la presencia de la falla Cerritos, denotada en las anomalías del CMRP y del filtro pasa-bajas por una transición de máximo a mínimo, y al contrario para la anomalía correspondiente al gradiente horizontal (Figura S3).

\section{Sísmica de refracción}

El modelo de velocidades obtenido presenta un rango limitado de alcance en profundidad $(60 \mathrm{~m})$ comparado con los otros métodos empleados. Sin embargo, los resultados muestran información detallada del subsuelo somero. En la Figura 9 se ilustra la distribución de velocidades de onda P (Vp) para el perfil ST. La Figura 9a muestra las celdas que indican la resolución vertical y horizontal del modelo de velocidades. A menor tamaño de las celdas, los cambios de velocidad son más precisos, por lo tanto, los primeros $10 \mathrm{~m}$ de profundidad en el modelo presentan la mejor resolución de velocidades para el perfil. Es importante mencionar que el tamaño de las celdas en las capas más profundas no varía considerablemente entre ellas, lo que indica que el ajuste de los datos es continuo a lo largo del modelo. Para este modelo, los valores de Vp varían entre $1900 \mathrm{~m} / \mathrm{s}$ en la base y $800 \mathrm{~m} / \mathrm{s}$ en las capas más someras. Los valores de velocidades aparentes y espesores promedio por capa se resumen en la Tabla 3.

En la Figura 9b se muestra el modelo suavizado de velocidad; aquí se observa que el gradiente de las velocidades se mantiene constante desde el extremo NO hasta una distancia de $120 \mathrm{~m}$ sobre el perfil. Tras superar esta distancia, se observan cambios laterales y abruptos de velocidad (indicados por las líneas discontinuas en color negro)

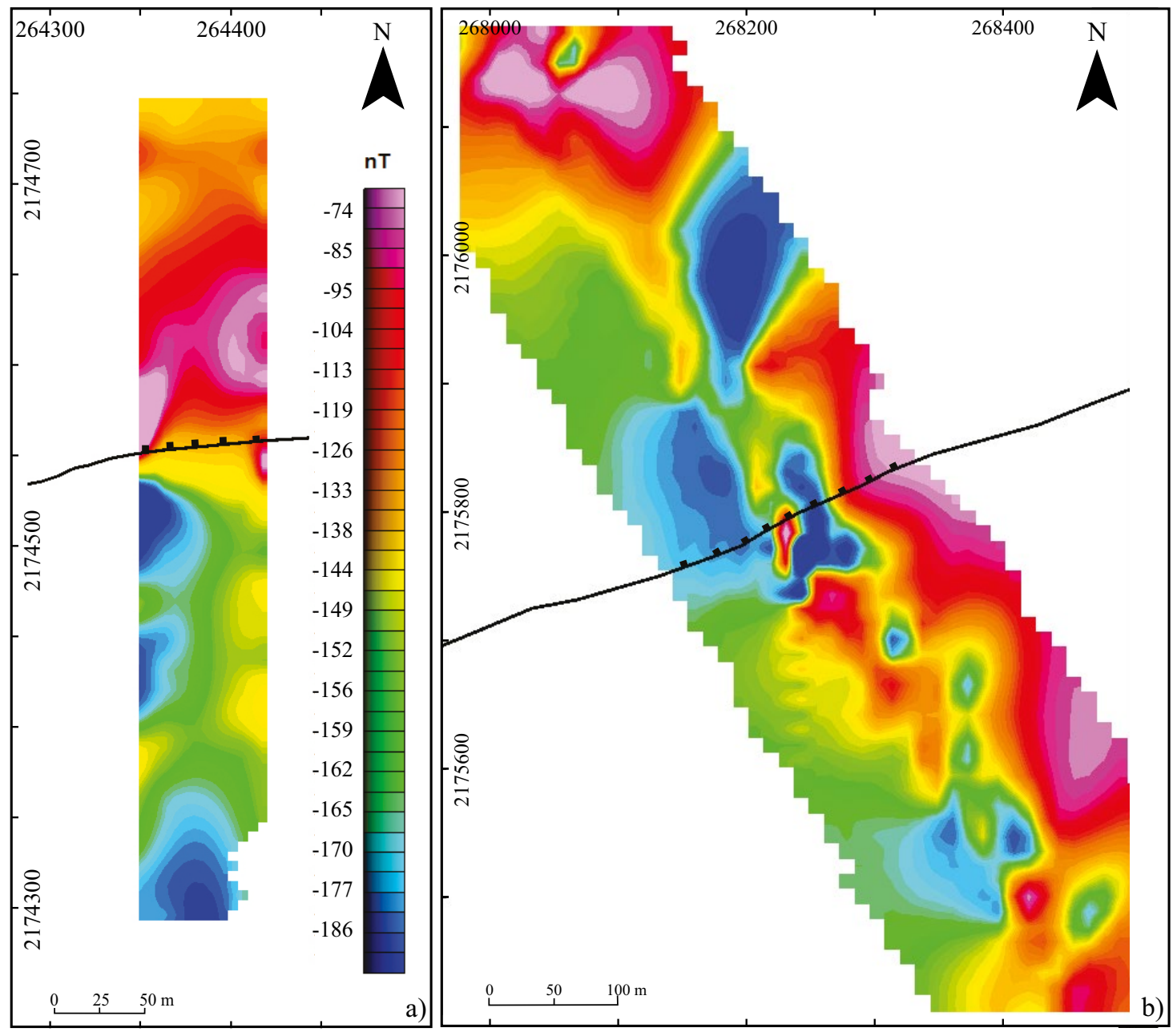

Figura 8. Mapa que muestra la configuración del campo magnético residual reducido al polo para el sector occidental (a) y oriental (b) de la falla Cerritos (ver perfiles en la Figura 3a y el sitio 1 en la Figura 5). 

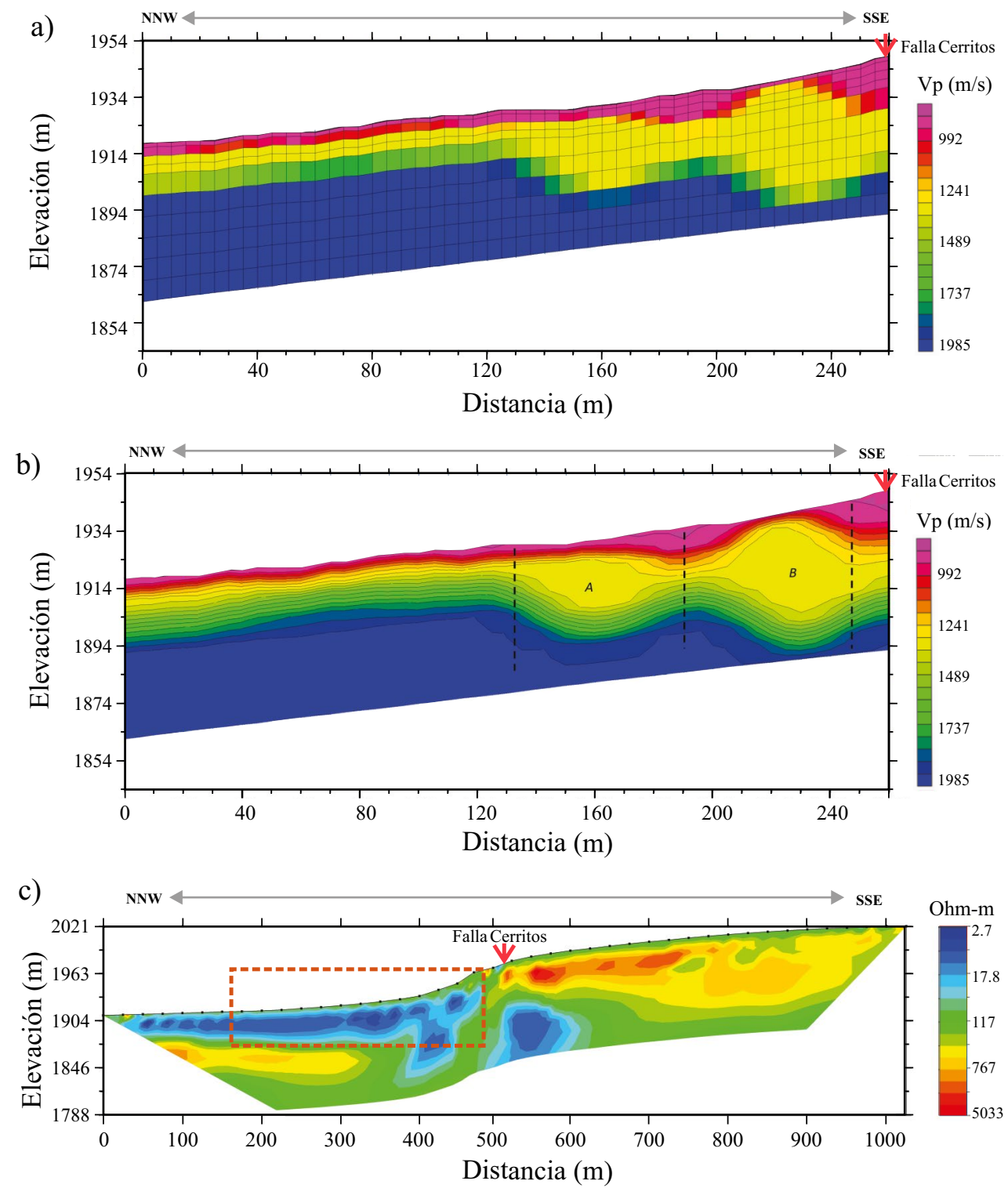

Figura 9. Resultado de los perfiles de sísmica y tomografía eléctrica en el sitio 1 (Figuras 3a y 5b). a) Modelo de velocidad de ondas P obtenido mediante sísmica de refracción para la falla Cerritos. Se muestran las celdas de resolución para el modelo obtenido indicando la resolución de las velocidades en cada capa. b) Modelo suavizado de distribución de velocidades de ondas sísmicas. Se indican los principales cambios laterales de velocidad con líneas segmentadas de color negro, y las zonas de disminución abrupta de velocidad con las letras $A$ y B. c) Perfil de Tomografía eléctrica sin interpretar, mostrando la distribución de las unidades de la resistividad eléctrica 1 (tonos cálidos y valores Ohm-m más altos) y 2 (tonos fríos y valores Ohm-m más bajos). El recuadro con línea punteada naranja representa la localización del perfil de sísmica de refracción. Las escalas vertical y horizontal son distintas para mejorar la visibilidad de los modelos.

en valores que oscilan alrededor de $400 \mathrm{~m} / \mathrm{s}$ en $5 \mathrm{~m}$ de distancia horizontal. Adicionalmente, se aprecian dos zonas de baja velocidad en una capa intermedia, con espesores de capas entre $20 \mathrm{~m} \mathrm{(A)} \mathrm{y} 30 \mathrm{~m}$ $(B)$, aproximadamente.

\section{Tomografía de Resistividad Eléctrica}

El perfil de tomografía obtenido tuvo una profundidad de investigación de $123 \mathrm{~m}$ y muestra dos claros contrastes en valores de resistividad real del subsuelo, los cuales fueron denominados como Unidades Geoeléctricas 1 y 2 (UG1 y UG2). La UG1 presenta valores de 478 a $5033 \mathrm{Ohm} \cdot \mathrm{m}$ (tonos cálidos, Figura 9c) y la UG2 valores de 2.7 a $45 \mathrm{Ohm} \cdot \mathrm{m}$ (tonos fríos). Se observa cómo ambas unidades pierden continuidad horizontal y tienen un desplazamiento vertical, entre la
Tabla 3. Velocidad aparente y espesor promedio por capa para el perfil sísmico.

\begin{tabular}{lcccc}
\hline Propiedad & Capa & Norte & Centro & Sur \\
\hline $\mathrm{Vp}(\mathrm{m} / \mathrm{s})^{*}$ & 1 & 865 & 875 & 698 \\
& 2 & 1265 & 1440 & 1183 \\
& 3 & 1805 & 2010 & 1840 \\
Espesor (m) & 1 & 1.4 & 3.3 & 1.8 \\
& 2 & 8.1 & 10.2 & 6.9 \\
& 3 & $>25.5$ & $>21.5$ & $>26$ \\
\hline
\end{tabular}

Nota: ${ }^{\star}$ Vp, velocidad aparente de las ondas P para el perfil de sísmica de refracción en la falla Cerritos. 
posición 300 y $492 \mathrm{~m}$ del perfil, que corresponde a la zona donde se empieza a evidenciar morfológicamente el escarpe de la falla Cerritos. La UG1 incluye los materiales con mayor densidad que se observan de manera dominante en la parte superior del bloque levantado del perfil, dichos materiales se corresponden con la ignimbrita Atécuaro de estructura masiva y bien soldada (Figura 9). En cambio, la UG2 incluye los materiales con menor densidad que se observan distribuidos en el bloque caído del perfil, abarcando los materiales depositados en el pie del escarpe. Dichos materiales podrían corresponder a ignimbritas altamente diaclasadas, depósitos de bloques caídos, secuencias lacustres y lahares. En el perfil se puede observar que el desplazamiento vertical de la UG2 es de $130 \pm 5 \mathrm{~m}$ (lo cual corresponde al salto total de la falla Cerritos).

\section{DISCUSIÓN}

\section{Geomorfología y tectónica activa}

El estudio de los índices geomórficos es útil para evaluar la tectónica activa de los sistemas de fallas. En ambientes extensionales, surgen rasgos característicos como frentes montañosos lineales formados por el hundimiento del bloque caído respecto al bloque levantado, y el desarrollo de un sistema de cuencas sensibles a la tectónica activa regional (Keller y Pinter, 1996; Özkaymak, 2015; Tepe y Sözbilir, 2017; He et al., 2018).

Los índices geomórficos asociados a la actividad tectónica de la zona de falla Cerritos muestran que cinco de las siete cuencas presentan una forma muy alargada a alargada y dos son ligeramente alargadas (Figura 6). La geometría larga y estrecha de las cuencas indica tasas elevadas de actividad tectónica relativamente reciente (Keller y Pinter, 1996; Tepe y Sözbilir, 2017). Seis de las cuencas presentan curvas hipsométricas convexas y sólo una cuenca se comporta como curva en forma de S (cuenca C3; Figura S2), sin embargo, todas presentan valores altos de integral hipsométrica $(I H>0.5)$, lo que sugiere un relieve muy joven con erosión a corto plazo, de manera análoga a otros casos reportados en el mundo (p.ej., Hurtezet al., 1999; Keller y Pinter, 1996; Tepe y Sözbilir, 2017).

La comparación de los valores hipsométricos y la forma de las cuencas muestra que C1, C3, C4 y C6 presentan poco desarrollo y superficies débilmente erosionadas (Figuras 6 y S2). Esto puede deberse tanto al factor tectónico como al factor litológico, ya que las cuencas se desarrollan sobre la ignimbrita Atécuaro, la cual tiene un grado de soldamiento alto. Sin embargo, dicha ignimbrita puede exhibir erosión diferencial asociada al fallamiento y a los cambios de facies con alta concentración de pómez y líticos, y baja proporción de matriz, lo cual es típico de los depósitos relacionados a corrientes de densidad piroclástica concentradas. Cabe resaltar que las curvas hipsométricas de las cuencas analizadas reflejan su desarrollo propio y no son influenciadas por la forma volcánica de la caldera de Atécuaro, ya que la región estudiada sólo ocupa la porción norte de la caldera.

Con base en el factor de asimetría se establece que las cuencas fluyen hacia el NNO, lo que indica que han estado influenciadas por la inclinación tectónica activa reciente, con una dirección de basculamiento del bloque levantado hacia el sureste. La dirección al NNO de las cuencas también podría estar relacionada con la paleo topografía de la caldera de Atécuaro. Asimismo, se puede ver que seis de las siete cuencas son dominantemente $V f<1$ en la relación ancho-alto, señal de que el bloque levantado con respecto al bloque caído es tectónicamente activo.

Los valores de $V f$ para las cuencas que se ubican al noreste tienen valores más cercanos o igual a 0 (C5 a C7), provocando la formación de valles en $\mathrm{V}$ con una mayor disección vertical (Figura 6), lo que sugiere que esta parte de la zona de falla es mucho más activa (p.ej., Keller y Pinter, 1996; Özkaymak, 2015; Tepe y Sözbilir, 2017). Por lo tanto, las características geomórficas de las cuencas en la zona de falla Cerritos (alargadas, poco espaciadas y ortogonales al escarpe) revelan un desarrollo topográfico reciente, provocado por el movimiento de esta falla.

La zona de falla de Cerritos es un frente de montaña que no presenta facetas triangulares ni trapezoidales, pero si un escarpe continuo, con pendientes entre $45^{\circ}$ y $85^{\circ}$. Esta morfología se ha interpretado como la expresión de fallas muy jóvenes y activas (Wallace, 1978; Keller y Pinter, 1996; Tepe y Sözbilir, 2017). Estas condiciones son respaldadas por el valor de 1.06 de la sinuosidad del frente de montaña, y la carencia de depósitos característicos como pie de monte o abanicos aluviales. Aunque la ausencia de abanicos aluviales podría deberse a la alta modificación antrópica del terreno, por lo que este criterio debe tomarse con precaución. De acuerdo con estudios mundiales, los valores de $S f m<1.4$ son indicativos de frentes tectónicos activos, donde el levantamiento prevalece sobre los procesos erosivos, dando como resultado frentes rectos con valores bajos de Sfm (Silva et al. 2003; Pérez-Peña et al., 2010).

\section{Estructura del subsuelo en la zona de falla}

Las anomalías magnéticas podrían indicar tanto cambios litológicos como zonas de debilidad en la corteza (Figura 8 y Figura S3). Las anomalías asociadas a bajos magnéticos (azules) podrían indicar la presencia de sedimentos fluvio-lacustres, de bloques caídos, o de la ignimbrita Atécuaro altamente alterada o diaclasada (Henkel y Guzmán, 1977; López-Loera et al., 2011). Mientras que las anomalías asociadas a altos magnéticos (rojos) podrían indicar la presencia de la ignimbrita Atécuaro sana, bien soldada y con estructura masiva (Figura 8).

La sísmica de refracción y tomografía de resistividad eléctrica permitieron caracterizar la geometría de la falla Cerritos en profundidad y su zona de deformación en su parte central-oriental. El espesor de la zona de falla es de aproximadamente 50 a $75 \mathrm{~m}$ en la horizontal (Figura 10c). Destaca la falla principal con una geometría aparentemente lístrica en profundidad, pero plana en superficie, que delimita una zona de desprendimiento. Sobre el plano de falla principal se interpretó la presencia de bloques caídos rotados y cortados por fallas sintéticas normales con geometría en dominó hacia el norte. El basculamiento desarrollado por la geometría de la falla pudo favorecer la formación de zonas tectónicamente inestables y anticlinales tipo roll-over, los cuales no mostrarían expresión superficial debido a la actividad humana en la zona y bajo la superficie pudieran estar representados por dos grabens de colapso crestal que funcionarían como depocentros de materiales clásticos y bloques caídos (Figura 10b). Otra interpretación sugiere que los depocentros al pie del escarpe pudieran relacionarse a fallas sintéticas secundarias al plano principal, al basculamiento propio del bloque caído y a la reología de los materiales involucrados; sin embargo, se requieren más perfiles a lo largo del escarpe para clarificarlo. Junto con la falla normal principal, con buzamiento hacia el norte (sintética), se generaron varias fallas secundarias antitéticas y conjugadas en el bloque caído, que se relacionan con la actividad de la falla primaria. Estas estructuras secundarias son fallas cuya expresión superficial no logró ser detectada por las modificaciones antrópicas del sitio, pero que se pudieran conectan dentro de la zona de deformación. Este arreglo estructural suele ser típico de ambientes extensionales y fallas normales (p.ej., Williams y Vann, 1987; Dula, 1991). Todas las unidades interpretadas en el perfil sísmico y eléctrico están deformadas por las fallas sintéticas y antitéticas, siendo la más evidente la ignimbrita Atécuaro, que acumula al menos $130 \pm 5 \mathrm{~m}$ de salto en la vertical (Figura 10c). Sin embargo, debemos considerar que las incertidumbres están asociadas a la insuficienteresolución de la sísmica de refracción para interpretar con certeza las estructuras más pequeñas en las zonas de 

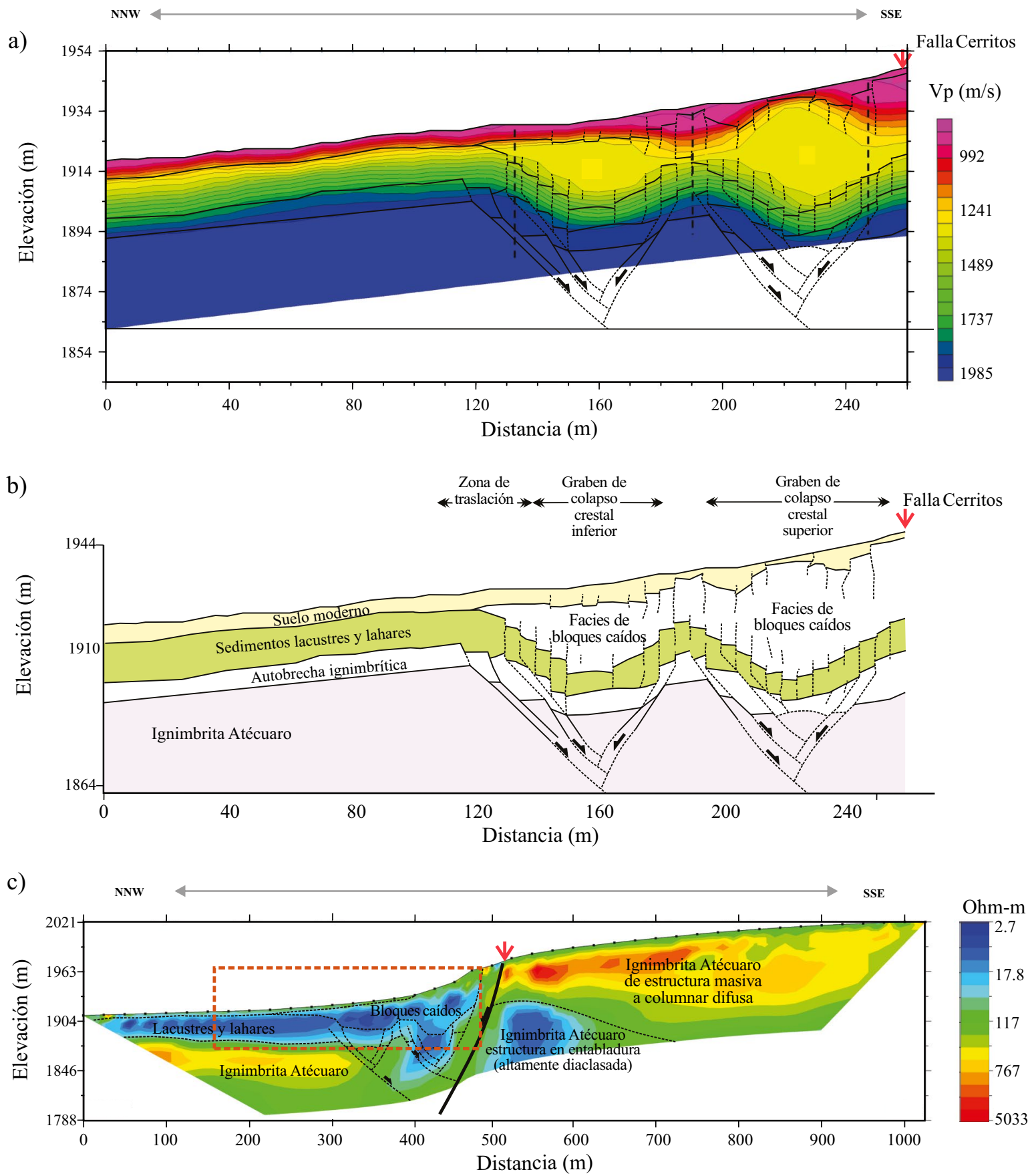

Figura 10. Interpretación del levantamiento geofísico. a y b) Sísmica de refracción. c) Tomografía de resistividad eléctrica (falla sintética principal marcada con línea más gruesa). El rectángulo con línea punteada naranja representa la ubicación del perfil de sísmica de refracción. Las escalas vertical y horizontal son distintas para mejorar la visibilidad de los modelos.

colapso; también cabe resaltar que la interpretación aquí propuesta requiere de la realización de más trabajo geofísico en la traza de la falla Cerritos para ser mejorada.

\section{Cinemática y deformación}

Las características geométricas y cinemáticas de la falla Cerritos confirman su origen tectónico. Su orientación ENE-OSO resulta de una extensión regional con dirección $\mathrm{NNO}\left(343^{\circ}\right)$ con una ligera componente lateral izquierda (Figura 5). Se encuentra óptimamente orientada con el campo de esfuerzos actual (Suter et al., 2001), y sus características son concordantes con las fallas del SFMA (GarduñoMonroy et al., 2009a; Suter, 2016; Mendoza-Ponce et al., 2018; GómezVasconcelos et al., 2021). Las familias de fallas NNO y NE en el área de estudio (Figuras 4 y 5) se encuentran estrechamente relacionadas con la falla Cerritos, ya que éstas comparten el mismo campo de esfuerzos, actuando como estructuras de Riedel R' y R, respectivamente, a causa de su ligera componente lateral izquierda.

En los extremos de la falla se aprecia un ligero cambio de rumbo de ENE a NE, asemejando una geometría sigmoidal, que junto con su arreglo en échelon (escalones hacia la derecha), nos revela una cinemá- 
tica oblicua-izquierda, similar a lo reportado en la evolución de otras fallas de la región (p.ej., Cembrano et al., 2005).

A pesar de que no hay registros de sismicidad histórica ni instrumental para la falla Cerritos y de que su escarpe principal corta la ignimbrita Atécuaro de 16.4 Ma, las observaciones realizadas en este trabajo sugieren la actividad reciente de esta falla. Los materiales desplazados por fallas en el afloramiento sugieren un último movimiento ocurrido hace menos de 3.37-3.21 cal ka AP (Tabla 2).

La magnitud potencial estimada para la falla Cerritos con base en su longitud fue de $M_{\mathrm{W}} 6.2$ a 6.6, lo cual se encuentra dentro de los valores reportados por Garduño-Monroy et al. (2009a; $M_{\mathrm{W}} 5.9$ a 6.5) y Gómez-Vasconcelos et al. (2021; $M_{\mathrm{W}} 6.2$ a 6.6). El tiempo de recurrencia promedio se estimó en 16700 años a partir de las estimaciones de desplazamiento esperado por evento y tasa de desplazamiento a largo plazo (ver abajo y Tabla 4).

De acuerdo con los resultados obtenidos en este trabajo y considerando un desplazamiento vertical máximo de $130 \pm 5 \mathrm{~m}$ en la ignimbrita Atécuaro ( $100 \mathrm{~m}$ de desplazamiento visible $+30 \mathrm{~m}$ de desplazamiento subsuperficial obtenido por el estudio de resistividad invertida; desplazamiento neto de $150 \mathrm{~m}$ ), podemos calcular una tasa de desplazamiento de $0.03 \pm 0.01 \mathrm{~mm} /$ año (largo plazo).

Aunque esta falla ya ha sido previamente identificada y existen datos que sugieren su actividad (p.ej., Garduño-Monroy et al., 2009a; Figueroa-Miranda et al., 2020; Gómez-Vasconcelos et al., 2021), los resultados del presente trabajo aportan nuevos parámetros sobre su estilo y geomorfología. Para la falla Cerritos, trabajos previos calcularon una tasa de desplazamiento promedio de $0.009 \mathrm{~mm} / \mathrm{año}$ (tasa a largo plazo; Garduño-Monroy et al., 2009a) hasta $0.03 \mathrm{~mm} /$ año (interpolada para el último millón de años; Gómez-Vasconcelos et al., 2021) La tasa de $0.009 \mathrm{~mm} /$ año determinada en publicaciones anteriores parece errónea, esto debido a que la edad del marcador que se utilizaba era una edad relativa $(5.3 \mathrm{Ma})$ y actualmente existe una edad absoluta (16.4 Ma); además, los insumos cartográficos usados en dicha publicación tenían menor resolución que los empleados en este trabajo, lo que resultó en una subestimación del salto de la falla. Cabe notar que los parámetros de la falla Cerritos son similares a los de sus fallas vecinas más estudiadas; Morelia y Cointzio, como lo son: longitud (12-15 km), desplazamiento promedio por evento $(0.5 \mathrm{~m}$ basado en las relaciones empíricas resueltas en este trabajo, Tabla 4) y $M_{\mathrm{W}}$ estimada (6.2-6.7; Gómez-Vasconcelos et al., 2021 y este estudio).

\section{Implicaciones para el peligro sísmico}

El crecimiento exponencial y poco planificado de Morelia ha incrementado la vulnerabilidad de sus habitantes ante riesgos sísmicos. Un evento de ruptura, tanto de la falla Cerritos como de cualquier otra de las fallas de esta ciudad, la impactaría notablemente, particularmente a las zonas cercanas a las fallas y a las construcciones asentadas sobre sedimentos lacustres debido a los fenómenos de amplificación de las ondas sísmicas (p.ej., Reinoso, 1995). Las características morfológicas de la falla Cerritos pueden relacionarse con inestabilidad de taludes y procesos de remoción en masa que afectarían a los asentamientos más cercanos (p.ej., colonias San José del Cerrito, La Floresta, Colinas del Sur, Vista Bella, Zimpanio Norte y Tenencia Morelos; Figura 5). Es conveniente realizar más estudios con el objetivo de tener una buena evaluación de la vulnerabilidad y el riesgo sísmico, lo cual permitirá a los pobladores buscar medidas de mitigación y protección adecuadas a su estilo de vida.

Es importante mencionar que la ciudad de Morelia también es afectada por subsidencia diferencial debido a procesos de creep detonados por la sobreexplotación de acuíferos, lo cual ha ocasionado daños a las obras civiles (Garduño-Monroy et al., 2001; Farina et al., 2008; Cigna et al., 2012). Para la falla Cerritos se calcula una tasa de desplazamiento de 0.49 a $0.99 \mathrm{~cm} /$ año asociado al movimiento creep y sus efectos son notorios en calles y construcciones cercanas a la falla (Figueroa-Miranda et al., 2020; mediciones de los períodos 2003-2010 y 2014-2017).

El desplazamiento asimétrico a lo largo de la falla Cerritos (Figura 5c) sugiere que esta falla está interactuando con fallas secundarias o vecinas, transfiriendo desplazamiento al traslaparse entre sí, siguiendo una evolución típica de ambientes extensivos (p.ej., Peacock, 2002; Nicol et al., 2010). La geometría sugiere que esta falla funciona como una rampa de relevo que la enlaza con las fallas Morelia y Cointzio, subparalelas entre sí (e.g., Suter, 2016). Es notable que la falla Cerritos presenta menor desplazamiento y longitud que las fallas adyacentes Morelia y Cointzio, lo que sugiere que es más joven que éstas. En términos de peligro sísmico, esto implica que la falla Cerritos podría romper junto con las fallas Cointzio, Morelia y Jaripeo, lo cual aumenta la peligrosidad sísmica de este conjunto de fallas al incrementarse la magnitud de los sismos que se producirían (hasta $M_{\mathrm{W}} 6.8$ y 6.9; Tabla 4). Este comportamiento de actividad multi-falla también es sugerido por el desplazamiento medido en la ruptura observada en

Tabla 4. Peligro sísmico asociado a la falla Cerritos.

\begin{tabular}{|c|c|c|c|c|c|c|c|c|}
\hline$\#$ & Falla & $\begin{array}{c}\text { Última } \\
\text { ruptura } \\
(\mathrm{Ma})\end{array}$ & $\begin{array}{l}\text { Longitud en } \\
\text { superficie } \\
(\mathrm{km})\end{array}$ & $\begin{array}{c}\text { Tasa desplaza- } \\
\text { miento } \\
(\mathrm{mm} / \mathrm{año})\end{array}$ & $\begin{array}{l}\text { Desplazamiento } \\
\text { por evento } \\
(\mathrm{m})\end{array}$ & $\begin{array}{l}\text { Intervalo de } \\
\text { recurrencia } \\
\quad(\mathrm{ka})\end{array}$ & $\begin{array}{c}\text { Magnitud } \\
\left(M_{w}\right) \text { Wells y } \\
\text { Coppersmith, } 2004\end{array}$ & $\begin{array}{c}\text { Magnitud }\left(M_{\mathrm{w}}\right) \\
\text { Wesnousky, } \\
2008\end{array}$ \\
\hline 1 & Cerritos & 0.003 & $12^{z}$ & 0.03 & 0.5 & 16.7 & 6.2 & 6.6 \\
\hline 2 & Morelia (Paloma) & $0.001^{\mathrm{x}}$ & 15 & 0.06 & 0.5 & 8.8 & 6.4 & 6.7 \\
\hline 3 & Cointzio & 0.62 & 15 & 0.06 & 0.5 & 8.3 & 6.4 & 6.7 \\
\hline 4 & Jaripeo & 3.6 & 15 & 0.03 & 0.5 & 16.7 & 6.4 & 6.7 \\
\hline $1+2$ & ${ }^{\star}$ Cerritos + Morelia & ND & 25 & 0.03 & 0.8 & 26.7 & 6.7 & 6.8 \\
\hline $1+2+3$ & ${ }^{\star}$ Cerritos+Morelia+Cointzio & ND & 35 & 0.03 & 1.2 & 40 & 6.9 & 6.8 \\
\hline $1+2+3+4$ & $\begin{array}{l}{ }^{*} \text { Cerritos+Morelia+Cointzio } \\
+ \text { +Jaripeo }\end{array}$ & ND & 50 & 0.03 & 1.7 & 56.7 & 7.1 & 6.9 \\
\hline
\end{tabular}

Nota: Magnitud momento $\left(M_{\mathrm{w}}\right)$ calculada con base en las ecuaciones de Wells y Coppersmith (2004) y Wesnousky (2008), para cierta longitud de ruptura superficial $(\mathrm{km})$ y área de falla. ${ }^{\approx}$ Tasa de desplazamiento promedio calculada a partir del desplazamiento neto en una superficie con edad conocida (ver Métodos).

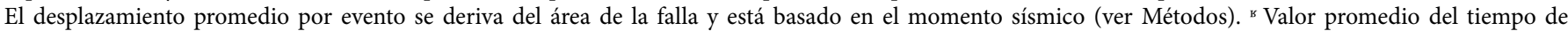
recurrencia ( área de una ruptura de falla debe ser igual o mayor a 1 (Wells y Coppersmith, 1994; Stirling et al., 2013). ${ }^{*}$ Rupturas potenciales en cadena (multi-falla). y Última ruptura tomada de estudios previos (Garduño-Monroy et al., 2001; Suter et al., 2001). ND, información no disponible. 
afloramiento para esta falla $(0.84 \pm 0.18 \mathrm{~m})$, la cual, de acuerdo con las relaciones empíricas, corresponde al desplazamiento promedio esperado para una ruptura de $25 \mathrm{~km}$ de largo y a un sismo con magnitud de $M_{\mathrm{W}} 6.7-6.8$, esto es equivalente a una ruptura conjunta de las fallas Cerritos + Morelia (Tabla 4).

\section{CONCLUSIONES}

El estudio y caracterización de la falla Cerritos representa una nueva contribución para el inventario de las fallas potencialmente sísmicas de la ciudad de Morelia y del Sistema de Fallas Morelia-Acambay en la evaluación del peligro sísmico.

La falla Cerritos es una falla normal con una ligera componente lateral izquierda, con geometría en échelon, longitud de $12 \mathrm{~km}$, dirección $255^{\circ}$, inclinación de $76^{\circ}$ hacia el NNO y produce un escarpe acumulado máximo de $130 \pm 5 \mathrm{~m}$ (desplazamiento neto de $150 \mathrm{~m}$ ) en la ignimbrita Atécuaro. Los análisis geológicos, geomorfológicos y estructurales indican que la falla Cerritos es tectónicamente activa, joven y con mayor actividad en el sector oriental.

El análisis geofísico muestra la estructura subsuperficial de la falla, resaltando la presencia de un plano principal lístrico y una zona de deformación en el bloque caído que se extiende de 50 a $75 \mathrm{~m}$ a partir del escarpe principal. Esta zona de deformación se caracteriza por la probable formación de anticlinales tipo roll-over y dos grabens de colapso crestal delimitados por fallas secundarias sin expresión superficial, que sirven como depocentros de materiales clásticos y bloques caídos. Aunque cabe resaltar que esta interpretación puede ser mejorada o modificada con trabajo posterior en la zona.

Para la falla Cerritos se estima una tasa de desplazamiento de $0.03 \pm 0.01 \mathrm{~mm} /$ año y un tiempo de recurrencia promedio de 16700 años. Con el último evento registrado ocurrido hace menos de 3.37$3.21 \mathrm{cal} \mathrm{ka} \mathrm{AP.} \mathrm{Esta} \mathrm{falla} \mathrm{tiene} \mathrm{una} \mathrm{magnitud} \mathrm{potencial} \mathrm{de} M_{\mathrm{W}} 6.2 \mathrm{a}$ 6.6; sin embargo, puede interaccionar con las fallas Morelia y Cointzio produciendo rupturas multi-falla, lo cual incrementa su peligrosidad al ser capaces de generar sismos de hasta $M_{\mathrm{W}} 6.8-6.9$ y desplazamientos promedio por evento de 0.5 a $1.2 \mathrm{~m}$ (para una ruptura individual y concatenada, respectivamente).

\section{AGRADECIMIENTOS}

Agradecemos a Isabel Israde, Sergio Nájera, Fidel Gómez, Julio Cañas, y Joaquín Gómez por su apoyo en las campañas de campo. Igualmente, agradecemos a los editores de este número especial (I. Israde y J.L. Macías) y a los revisores de este trabajo (M. Ortuño Candela y un revisor anónimo) por su tiempo y comentarios para mejorar este trabajo.

Agradecemos al Consejo Nacional de Ciencia y Tecnología (CONACYT) a través del proyecto A1-S-23296 a D.R. Avellán, y del proyecto p.17 de CEMIE-Geo a V.H. Garduño. M.G. Gómez agradece a la Beca para las mujeres en la ciencia L' Oréal-UNESCO-AMC 2021.

El presente trabajo está dedicado al Dr. Víctor Hugo Garduño Monroy, cuya dedicación y conocimiento fueron elementos cruciales en la formación profesional de los autores y de tanta gente.

\section{SUPLEMENTO ELECTRÓNICO}

Las Figuras S1, S2 y S3, así como las Tablas S1 y S2 están disponibles en el portal web de esta Revista <www.rmcg.unam.mx>, dentro de la hoja de presentación previa de este artículo.

\section{REFERENCIAS}

Abe, K., 1981, Magnitudes of large shallow earthquakes from 1904 to 1980: Physics of the Earth and Planetary Interiors, 27 (1), 72-92.

Andreani, L., Le Pichon, X., Rangin, C., Martínez-Reyes, J., 2008, The southern Mexico block: main boundaries and new estimation for its Quaternary motion: Bulletin de la Société géologique de France, 179 (2), 209-223.

Arzate, J., Lacan, P., Corbo-Camargo, F., Arango-Galván, C., Félix-Maldonado, R., Pacheco, J., León-Loya, R., 2018, Crustalstructureoftheeastern Acambay Graben, Central Mexico, from integrated geophysical data: Revista Mexicana de Ciencias Geológicas, 35(3), 228-239, DOI: 10.22201/ cgeo.20072902e.2018.3.864

Astíz-Delgado, L.M., 1980, Sismicidad en Acambay, Estado de México. El temblor del 22 de febrero de 1979: México, D.F., México,Universidad Nacional Autónoma de México, Facultad de Ingeniería, Tesis profesional, $130 \mathrm{pp}$.

Avellán, D.R., Cisneros-Máximo, G., Macías, J.L., Gómez-Vasconcelos, M.G., Layer, P.W., Sosa-Ceballos, G., Robles-Camacho, J., 2020, Eruptive chronology of monogenetic volcanoes northwestern of Morelia - Insights into volcano-tectonic interactions in the central-eastern MichoacánGuanajuato volcanic field, México: Journal of South American Earth Sciences, 100, 102554.

Ávila-Olivera, J.A., Garduño-Monroy, V.H., 2008, A GPR study of subsidencecreep-fault processes in Morelia, Michoacán, Mexico: Engineering Geology, 100(1-2), 69-81.

Bull, W.B., 1977, Tectonic geomorphology of the Mojave Desert, California: Menlo Park, CA., US Geological Survey Contract Report 14-0-001-G-394, Office of Earthquakes, volcanoes, and engineering, $188 \mathrm{pp}$.

Bull, W.B., McFadden, L.D., 1977, Tectonic geomorphology north and south of the Garlock fault, California, en Doehering D.O. (ed.), Geomorphology in arid regions: Binghamton, NY, Proceedings at the Eightn Annual Geomorphology Sympossium, State University of New York., 115-138.

Camacho, H., 1925, Las aguas subterráneas del valle de Morelia, Estado de Michoacán: Anales del Instituto Geológico de México, 2, 6-17.

Carranza-Castañeda, O., 1976, Paleomastozoología de la cuenca de San Miguel de Allende, Estado de Guanajuato, sus contribuciones: el intercambio biótico entre las Américas: Paleontología Mexicana, 3(1), 73-83.

Cembrano, J., González, G., Arancibia, G., Ahumada, I., Olivares, V., Herrera, V., 2005, Fault zone development and strain partitioning in an extensional strike-slip duplex: A case study from the Mesozoic Atacama fault system, Northern Chile: Tectonophysics, 400(1-4), 105-125.

Chico Hernández, C. E., Ruiz Barón, D., 2017, Caracterización de sismos históricos de México a partir de valores de intensidad: Ciudad de México, México, Universidad Nacional Autónoma de México, Facultad de Ingeniería, Tesis de Ingeniería Geofísica, 103 pp.

Cigna, F., Osmanoğlu, B., Cabral-Cano, E., Dixon, T.H., Ávila-Olivera, J.A., Garduño-Monroy, V.H., DeMets, C. y Wdowinski, S., 2012, Monitoring land subsidence and its induced geological hazard with Synthetic Aperture Radar Interferometry: A case study in Morelia, Mexico: Remote Sensing of Environment, 117, 146-161.

Comisiones del Instituto Geológico de México, 1922, Memoria relativa al terremoto mexicano del 3 de enero de 1920: Instituto Geológico de México, Boletín 38, 106 pp.

Cortés-Arreola, R., 1985, Obras completas de D. Melchor Ocampo. V. 1. La obra científica y literaria: Morelia, Mich., México, Comité Editorial del Gobierno del Estado de Michoacán, 510 pp.

Demant, A., 1978, Características del Eje Neovolcánico Transmexicano y sus Problemas de Interpretación: Revista Instituto de Geología, Universidad Nacional Autónoma de México, 2(2), 172-187.

Dobson, P.F., Mahood, G.A., 1985, Volcanicstratigraphyofthe Los Azufres geothermalarea, Mexico: Journal of Volcanology and Geothermal Research, 25, 273-287.

Dula Jr., W.F., 1991, Geometric models of listric normal faults and rollover folds: AAPG Bulletin, 75(10), 1609-1625.

Ego, F., Ansan, V., 2002, Why is the Central Trans-Mexican Volcanic Belt $\left(102^{\circ}-99^{\circ} \mathrm{W}\right)$ in transtensive deformation?: Tectonophysics, 359, 189-208.

Farina, P., Ávila-Olivera, J.A., Garduño-Monroy, V.H., Catani, F., 2008, DInSAR analysis of differential ground subsidence affecting urban areas along the Mexican Volcanic Belt (MVB): Rivista Italiana di Telerilevamento (AIT), 
Il Telerilevamento a microonde, L'attività di ricerca e le applicazioni, 40 (2), 103-113

Ferrari, L., Pasquaré, G., Tibaldi, A., 1990, Plio-Quaternary tectonics of central Mexican Volcanic Belt and some constraints on its rifting mode: Geofísica Internacional, 29 (1), 5-18.

Ferrari, L., Orozco-Esquivel, T., Manea, V., Manea, M., 2012, The dynamic history of the Trans-Mexican Volcanic Belt and the Mexico subduction zone: Tectonophysics, 522, 122-149.

Figueroa-Miranda, S., Hernández-Madrigal, V.M., Tuxpan-Vargas, J., Villaseñor-Reyes, C.I., 2020, Evolution assessment of structurallycontrolled differential subsidence using SBAS and PS interferometry in an emblematic case in Central Mexico: Engineering Geology, 279, 105860.

Garduño, V.H., Alcalá, S., Mendoza, M.E., Hernández, V.M., 2007, Comportamiento histórico y actual del espejo de agua del lago de Pátzcuaro, en Ávila-García (coord.), Aguas y lagos de Michoacán: una mirada desde lo global hasta lo local: Morelia, Michoacán México, Secretaría de Cultura del Estado de Michoacán, Secretaría de Urbanismo y Medio Ambiente del Michoacán y Colegio de Michoacán, Biblioteca Michoacana, 157-170.

Garduño-Monroy, V.H., Gutiérrez-Negrín, L.C.A., 1992, Magmatismo, hiatus y tectonismo de la Sierra Madre Occidental y del Cinturón Volcánico Mexicano: Geofísica Internacional, 31(4).

Garduño-Monroy, V.H., Rocha, E.A., Israde-Alcántara, I., Torres, G.M.R., 2001, Efectos de las fallas asociadas a sobreexplotación de acuíferos y la presencia de fallas potencialmente sísmicas en Morelia, Michoacán, México: Revista Mexicana de Ciencias Geológicas, 18(1), 37-54.

Garduño-Monroy, V.H., Pérez-López, R., Israde-Alcántara, I., RodríguezPascua, M.A., Szynkaruk, E., Hernández-Madrigal, V.M., García-Zepeda, M.L., Corona-Chávez, P., Ostroumov, M., Medina-Vega, V.H., GarcíaEstrada, G., Carranza, O., López-Granados, E., Mora Chaparro, J.C., 2009a, Paleoseismology of the southwestern Morelia-Acambay fault system, central Mexico: Geofísica Internacional, 48(3), 319-335.

Garduño-Monroy, V. H., Chávez-Hernández, J., Aguirre-González, J., VázquezRosas, R., Mijares Arellano, H., Israde-Alcántara, I., Hernández-Madrigal, V.H., Rodríguez-Pascua, M.A., Pérez López, R., 2009b, Zonificación de los períodos naturales de oscilación superficial en la ciudad de Pátzcuaro, Mich., México, con base en microtremores y estudios de paleosismología: Revista Mexicana de Ciencias Geológicas, 26(3), 623-637.

Gómez-Tuena, A., Orozco-Esquivel, M.T., Ferrari, L., 2005, Petrogénesis ígnea de la faja volcánica transmexicana: Boletín de la Sociedad Geológica Mexicana, 57(3), 227-283.

Gómez-Vasconcelos, M.G., Garduño-Monroy, V.H., Macías, J.L., Layer, P.W., Benowitz, J.A., 2015, The Sierra de Mil Cumbres, Michoacán, México: Transitional volcanism between the Sierra Madre Occidental and TransMexican volcanic belt: Journal of Volcanology and Geothermal Research, 301, 128-147.

Gómez-Vasconcelos, M.G., Macías, J.L., Avellán, D.R., Sosa-Ceballos, G., Garduño-Monroy, V.H., Cisneros-Máximo, G., Layer, P.W., Benowitz, J., López-Loera, H., Mendiola López, F., Perton, M., 2020, The control of preexisting faults on the distribution, morphology, and volume of monogenetic volcanism in the Michoacán-Guanajuato Volcanic Field: GSA Bulletin, 132 (11-12), 2455-2474.

Gómez-Vasconcelos, M.G., Avellán, D.R., Soria-Caballero, D., Macías, J.L., Velázquez-Bucio, M.M., Jiménez-Haro, A., Israde-Alcántara I., GarduñoMonroy, V.H., Ávila-Olivera, J.A., Figueroa-Soto, Á., Cisneros-Máximo, G., Cardona-Melchor, S., 2021, Geomorphic characterization of faults as earthquake sources in the Cuitzeo Lake basin, central México: Journal of South American Earth Sciences, 109, 103196

Guzmán-Speziale, M., Zúñiga, F.R., 2016, Differences and similarities in the Cocos-North America and Cocos-Caribbean convergence, as revealed by seismic moment tensors: Journal of South American Earth Sciences, 71, 296-308.

Hare, P.W., Gardner, T.W., 1985, Geomorphic indicators of vertical neotectonism along converging plate margins, Nicoya Peninsula, Costa Rica, en Morisawa, M., Hack J.T. (eds.), Tectonic geomorphology: Boston, MA, EE.UU., Proceedings of the 15th Annual Bighamton Geomorphology Symposium, Allen and Unwin, 75-104.

He, C., Cheng, Y., Rao, G., Chen, P., Hu, J., Yu, Y., Yao, Q., 2018, Geomorphological signatures of the evolution of active normal faults along the Langshan Mountains, north China: Geodinamica Acta, 30 (1), 163-182.
Henkel, H., Guzmán, M., 1977, Magnetic features of fracture zones: Geoexploration, 15(3), 173-181.

Horton, R.E., 1932, Drainage basin characteristics: American Geophysical Union, Tr, 350-361.

Hurtez, J.E., Sol, C., Lucazeau, F., 1999, Effect of drainage area on hypsometry from an analysis of small-scale drainage basins in the Siwalik Hills (Central Nepal): Earth Surface Processes and Landforms, 24, 799-808.

INEGI (Instituto Nacional de Estadística y Geografía), 2020, Censo de Población y Vivienda, 2020, disponible en <www.inegi.org.mx/programas/ ccpv/2020/> accesado el 30 de enero de 2021.

Israde Alcántara, I., Velázquez-Durán, R., Lozano García, M., Bischoff, J., Domínguez Vázquez, G., Garduño Monroy, V.H., 2010, Evolución Paleolimnológica del Lago Cuitzeo, Michoacán durante el PleistocenoHoloceno: Boletín de la Sociedad Geológica Mexicana, 62(3), 345-357.

Keller, E.A., 1986, Investigation of active tectonics: Use of surficial Earth processes, en Wallace R.E. (ed.), Active tectonics: Washington, DC, EE.UU., National Academy Press, Studies in Geophysics, 136-147.

Keller, E.A., Pinter, N., 1996, Active tectonics. Earthquakes, uplift, and landscape: Upper Saddle River, NJ, Prentice Hall, 338 pp.

Lacan, P., Ortuño, M., Audin, L., Perea, H., Baize, S., Aguirre-Díaz, G., Zúñiga, F.R., 2018, Sedimentary evidence of historical and prehistorical earthquakes along the Venta de Bravo Fault System, Acambay Graben (Central Mexico): Sedimentary Geology, 365, 62-77.

Langridge, R.M., Weldon II, R.J., Moya, J.C., Suárez, G., 2000, Paleoseismology of the 1912 Acambay earthquake and the Acambay-Tixmadejé Fault, Trans-Mexican Volcanic Belt: Journal of Geophysical Research, Solid Earth, (1978-2012), 105 (B2), 3019-3037.

Libby, W.F., 1961, Radiocarbon dating: Science, 133(3453), 621-629.

Loke, M.H., 2015, Tutorial: 2D and 3D electrical imaging surveys: Geotomo Software, Malaysia.

López-Loera, H., Urrutia-Fucugauchi, J., Alva-Valdivia, L., 2011, Estudio aeromagnético del complejo volcánico de Colima, occidente de Méxicoimplicaciones tectónicas y estructurales: Revista Mexicana de Ciencias Geológicas, 28(3), 349-370.

Manea, V.C., Manea, M., Ferrari, L., 2013, A geodynamical perspective on the subduction of Cocos and Rivera plates beneath Mexico and Central America: Tectonophysics, 609, 10.1016/j.tecto.2012.12.039

Mendoza-Ponce, A., Figueroa-Soto, A., Soria-Caballero, D., Garduño-Monroy, V.H., 2018, Active faults sources for the Pátzcuaro-Acambay fault system (Mexico): fractal analysis of slip rates and magnitudes $M_{W}$ estimated from fault length: Natural Hazards and Earth System Sciences, 18(11), 3121-3135.

Mennella, L., 2011, Sismotectónica del sector occidental del sistema MoreliaAcambay, México, a partir del análisis de poblaciones de fallas: Morelia, Mich., México, Universidad Michoacana de San Nicolás de Hidalgo, Tesis de maestría, 151 pp.

Nicol, A., Walsh, J.J., Villamor, P., Seebeck, H., Berryman, K.R., 2010, Normal fault interactions, paleoearthquakes and growth in an active rift: Journal of Structural Geology, 32(8), 1101-1113.

Orozco y Berra, J., 1887, Efemérides sísmicas mexicanas: Memorias de la Sociedad Científica Antonio Alzate, 1, 303-637.

Ortuño, M., Zúñiga, F. R., Aguirre-Díaz, G. J., Carreón-Freyre, D., Cerca, M., Roverato, M., 2015, Holocene paleo-earthquakes recorded at the transfer zone of two major faults: The Pastores and Venta de Bravo faults (Trans-Mexican Volcanic Belt): Geosphere, 11(1), 160-184, doi: 10.1130/ GES01071.1

Ortuño, M., Corominas, O., Villamor, P., Zúñiga, R. F., Lacan, P., AguirreDíaz, G., Perea, H., Štěpančíková, P., Ramírez-Herrera, M.T., 2019 Evidence of recent ruptures in the central faults of the Acambay Graben (central Mexico): Geomorphology, 326, 17-37,doi: 10.1016/j. geomorph.2018.07.010

Özkaymak, Ç., Sözbilir, H., 2012, Tectonic geomorphology of the Spildağı high ranges, western Anatolia: Geomorphology, 173, 128-140.

Özkaymak, Ç., 2015, Tectonic analysis of the Honaz Fault (western Anatolia) using geomorphic indices and the regional implications: Geodinamica Acta, 27(2-3), 109-128.

Pardo, M., Suárez, G., 1995, Shape of the subducted Rivera and Cocos plates in southern Mexico: seismic and tectonic implications: Journal of Geophysical Research, 100 (B7), 12357-12373.

Pasquarè, G., Ferrari, L., Garduño, V.H., Tibaldi, A., Vezzoli, L., 1991, Geologic 
map of the central sector of the Mexican volcanic belt, states of Guanajuato and Michoacan, Mexico: Geological Society of America Map and Chart Series, $\mathrm{MCH} 072$

Peacock, D.C.P., 2002, Propagation, interaction and linkage in normal fault systems: Earth-Science Reviews, 58(1-2), 121-142.

Pérez-Peña, J.V., Azor, A., Azañon, J.M., Keller, E.A., 2010, Active tectonics in the Sierra Nevada (Betic Cordillera, SE Spain): Insights from geomorphic indexes and drainage pattern analysis: Geomorphology, 119, 74-87.

Quintanar, L., Rodríguez-González, M., Campos-Enríquez, O., 2004, A shallow crustal earthquake doublet from the Tran-Mexican Volcanic Belt (Central Mexico): Bulletin of the Seismological Society of America, 94, 845-855.

Ramírez, S., Reyes, V., 1873, Informe sobre los temblores de Agua Fría y Jaripeo: Boletín de la Sociedad Mexicana de Geografía y Estadística, época 3(1), 67-68.

Ramsey, C.B., 2009, Bayesian analysis of radiocarbon dates: Radiocarbon, 51(1), 337-360

Reimer, P.J., Austin, W.E., Bard, E., Bayliss, A., Blackwell, P.G., Ramsey, C.B., Butzin, M., Cheng, H., Edwards, R.L., Friedrich, M., Grootes, P.M., 2020, The IntCal20 Northern Hemisphere radiocarbon age calibration curve (0-55 cal kBP): Radiocarbon, 62(4), 725-757.

Reinoso, E., 1995, Características sísmicas de la ciudad de México: Amplificación de las ondas sísmicas en el Valle de México, características del movimiento del terreno y microzonificación de la ciudad, en Curso sobre seguridad sísmica de las construcciones para directores responsables de la obra: México, D.F., México, Centro Nacional de Prevención de Desastres (CENAPRED) 53-82.

Rodríguez-Pérez, Q., Zúñiga, F.R., 2017, Seismicitycharacterizationofthe Maravatío, Acambay and actopanregions, central Mexico: Journal of South American Earth Sciences, 76, 264-275.

Sánchez-Garcilazo, V., 2000, Estudio de la Macrosismicidad del Estado de Michoacán: Morelia, Mich., México, Universidad Michoacana de San Nicolás de Hidalgo, Facultad de Ingeniería Civil, Tesis de Maestría, 133 pp.

Silva, P.G., Goy, J.L., Zazo, C., Bardají, T., 2003, Fault-generated mountain fronts in southeast Spain: Geomorphologic assessment of tectonic and seismic activity: Geomorphology, 50, 203-225.

Singh, S.K., Ordaz, M., Pérez-Rocha, L., 1996, The great Mexican earthquake of 19 June 1858: Expected ground motions and damage in Mexico City from a similar future event: Bulletin of the Seismological Society of America, 86(6), 1655-1666.

Singh, S.K., Iglesias, A., Garduño, V.H., Quintanar, L., Ordaz, M., 2012, A source of the October, 2007 earthquake sequence of Morelia, Mexico and ground-motion estimation from larger earthquakes in the region: Geofísica Internacional, 5 (1), 73-86.

Soria-Caballero, D.C, Garduño-Monroy, V.H, Alcalá, M., Velázquez-Bucio, M.M, Grassi, L, 2019, Evidence for Quaternary seismic activity of the La Alberca-Teremendo fault, Morelia region, Trans-Mexican Volcanic Belt: Revista Mexicana de Ciencias Geológicas, 36(2), 242-258.

Stirling, M., Goded, T., Berryman, K., Litchfield, N., 2013, Selection of earthquake scaling relationships for seismic-hazard analysis: Bulletin of the Seismological Society of America, 103(6), 2993-3011.

Suárez, G., Caballero-Jiménez, G.V., Novelo-Casanova, D.A., 2019, Active crustal deformation in the Trans-Mexican volcanic belt as evidenced by historical earthquakes during the last 450 years: Tectonics, 38(10), 3544-3562.

Sunye-Puchol, I., Lacan, P., Ortuño, M., Villamor, P., Audin, L., Zúñiga, F. R., Langridge, R.M., Aguirre-Díaz, G.J., Lawton, T.F., 2015, La falla San Mateo: nuevas evidencias paleosismológicas de fallamiento activo en el graben de Acambay, México: Revista Mexicana de Ciencias Geológicas, 32(3), 361-376.
Suter, M., 2014, Comment on "Estimation of ground motion in Mexico City from a repeat of the $M \sim 7.0$ Acambay Earthquake of 1912" by S.K. Singh, A. Iglesias, M. Ordaz, X. Pérez-Campos, and L. Quintanar: Bulletin of the Seismological Society of America, 104(5), 2562-2564.

Suter, M., 2016, Structure and Holocene rupture of the Morelia fault, TransMexican volcanic belt, and their significance for seismic-hazard assessment: Bulletin of the Seismological Society of America, 106(5), 2376-2388.

Suter, M, 2017, The 2 October 1847 MI 5.7 Chapala Graben Triggered Earthquake (Trans-Mexican Volcanic Belt, West-Central Mexico): Macroseismic Observations and Hazard Implications: Seismological Research Letters, 89(1), 35-46, doi: 10.1785/0220170101

Suter, M., Quintero-Legorreta, O., Johnson, C.A., 1992, Active faults and state of stress in the central part of the Trans-Mexican Volcanic Belt, Mexico; 1, The Venta de Bravo Fault: Journal of Geophysical Research, 97, 11983-11993.

Suter, M., Quintero-Legorreta, O., López-Martínez, M., Aguirre-Díaz, G., Farrar, E., 1995, The Acambay graben: Active intraarcextension in the TransMexican Volcanic Belt, Mexico: Tectonics 14(6), 1245-1262.

Suter, M., López-Martínez, M., Quintero-Legorreta, O., Carrillo-Martínez, M., 2001, Quaternary intra-arc extension in the Central Trans-Mexican volcanic belt: Geological Society of America Bulletin, 113(6), 693-703.

Tepe, C., Sözbilir, H., 2017, Tectonic geomorphology of the Kemalpasa basin and surrounding horsts, southwestern part of the Gediz graben, western Anatolia: Geodinámica Acta, 29(1), 70-90.

Urbina, F., Camacho, H., 1913, La zona megasísmica Acambay- Tixmadejé, Estado de México, conmovida el 19 de noviembre de 1912: Boletín del Instituto Geológico de México, 32, 125 pp.

Urquiza, M., 1872, Informe del ingeniero encargado de reconocer la Sierra de Ucareo para averiguar las probabilidades de una erupción volcánica: Boletín de la Sociedad Mexicana de Geografía y Estadística, época 2(4), 586-588.

Wallace, R.E., 1978, Geometry and rates of change of fault-generated range fronts, north-central Nevada: Journal of Research of the US Geological Survey, 6, 637-650.

Wells, D.L., Coppersmith, K.J., 1994, New empirical relationships among magnitude, rupture length, rupture width, rupture area, and surface displacement: Bulletin of the seismological Society of America, 84(4), 974-1002.

Wesnousky, S.G., 2008, Displacement and geometrical characteristics of earthquake surface ruptures: Issues and implications for seismichazard analysis and the process of earthquake rupture: Bulletin of the Seismological Society of America, 98(4), 1609-1632.

Williams, G., Vann, I., 1987, The geometry of listric normal faults and deformation in their hanging walls: Journal of Structural Geology, 9(7), 789-795.

Manuscrito recibido: marzo 1, 2021

Manuscrito corregido recibido: agosto 17, 2021

Manuscrito aceptado: septiembre 29, 2021 